\title{
Exploring the nature of ambiguous merging systems: GW190425 in low latency
}

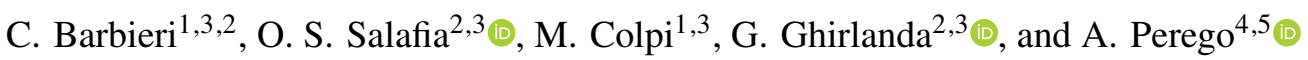 \\ 1 Università degli Studi di Milano-Bicocca, Dipartimento di Fisica “G. Occhialini”, Piazza della Scienza 3, 20126 Milano, Italy \\ e-mail: c.barbieri@campus .unimib.it \\ 2 INAF - Osservatorio Astronomico di Brera, Via E. Bianchi 46, 23807 Merate, Italy \\ 3 INFN - Sezione di Milano-Bicocca, Piazza della Scienza 3, 20126 Milano, Italy \\ 4 Università degli Studi di Trento, Dipartimento di Fisica, Via Sommarive 14, 38123 Trento, Italy \\ 5 INFN-TIFPA, Trento Institute for Fundamental Physics and Applications, Via Sommarive 14, 38123 Trento, Italy
}

Received 20 February 2020 / Accepted 2 July 2021

\begin{abstract}
GW190425 is a recently discovered gravitational wave (GW) source whose individual binary components are consistent with being neutron stars (NSs). However, the source-frame chirp mass $1.44 \pm 0.02 M_{\odot}$ is larger than that of any double NS system known as yet, and it falls in the 'ambiguous' interval for which the presence of a black hole $(\mathrm{BH})$ cannot be ruled out from the GW signal analysis alone. GW190425 might host an NS and a light BH, with a mass in the so-called lower mass gap. No electromagnetic (EM) counterpart has been associated with this event, due to the poorly informative sky localisation and larger distance compared to GW 170817 . We construct kilonova (KN) light curve models for GW190425, in both the double NS and BH-NS scenarios, considering two equations of state (EoSs) consistent with current constraints from GW170817 and the NICER results, including BH spin effects, and testing different fitting formulae for the ejecta mass. According to our models, the putative presence of a light BH in GW190425 would have produced a brighter $\mathrm{KN}$ emission compared to the double NS case, ideally leading to the possibility of distinguishing the nature of the binary. However, depending on the adopted fitting formula for the ejecta, the feasibility of this distinction might depend on the EoS and on the BH spin. Concerning candidate counterparts of GW190425, classified later on as supernovae, our models could have been used to discard two transients detected in their early $r$-band evolution, as these fall outside the phase space encompassed by our models. We conclude that combining the chirp mass and distance information from the GW signal with a library of KN light curves can help in identifying the EM counterpart early on, and we stress that the low-latency release of the chirp mass in this interval of ambiguous values can be vital for successful EM follow-ups.
\end{abstract}

Key words. stars: neutron - stars: black holes - binaries: general - gravitational waves

\section{Introduction}

The LIGO Scientific Collaboration and Virgo Collaboration (LVC) detected gravitational waves (GWs) from the inspiral and merger of several stellar origin black hole-black hole (BHBH) binaries (The LIGO Scientific Collaboration \& the Virgo Collaboration 2019a) during the observing runs O1 and O2 (2015-2017). In August 2017, the first neutron star-neutron star (NSNS) binary coalescence was detected (GW170817; The LIGO Scientific Collaboration \& the Virgo Collaboration 2017a), which was accompanied by a broadband electromagnetic (EM) counterpart (Abbott et al. 2017), heralding the birth of multi-messenger GW-EM astronomy. Recently, during the $\mathrm{O} 3$ run, the second NSNS merger was detected (GW190425; The LIGO Scientific Collaboration \& the Virgo Collaboration 2020), but no EM counterpart was firmly associated with this event ${ }^{1}$ (Coughlin et al. 2019).

The merger of a black hole-neutron star (BHNS) binary is a highly anticipated GW source (The LIGO Scientific Collaboration \& the Virgo Collaboration 2010). At the time of writing, LVC has reported promising candidates ${ }^{2}$,

\footnotetext{
1 Pozanenko et al. 2020 suggested an association with GRB190425, although Foley et al. (2020) and Song et al. (2019) showed that the data were not constraining in this sense.

2 A complete list of candidates is available on the LIGO/Virgo O3 Public Alerts webpage https://gracedb.ligo.org/superevents/ public/03/.
}

such as S190910d (The LIGO Scientific Collaboration \& the Virgo Collaboration 2019b) and S191205ah (The LIGO Scientific Collaboration \& the Virgo Collaboration 2019c), and has discovered GW190814, which is potentially the first BHNS detected. The case of GW190814 (Abbott et al. 2020) is particularly interesting as the source hosts a $22.2-24.3 M_{\odot}$ black hole $(\mathrm{BH})$ and a secondary compact object with a mass of 2.50-2.67 $M_{\odot}$ that could either be the lightest BH or the heaviest neutron star (NS) ever discovered. No EM counterpart was associated with these candidates, which hampered the possibility of shedding light on the nature of these systems (see The ENGRAVE Collaboration 2020; Coughlin et al. 2020, and references therein).

It is anticipated that BHNS mergers can produce EM counterparts as NSNS mergers do, depending on the combination of four binary parameters, namely the $\mathrm{BH}$ mass $M_{\mathrm{BH}}$ and $\operatorname{spin}^{3}$ $\chi_{\mathrm{BH}}$, the NS mass $M_{\mathrm{NS}}$, and tidal deformability $\Lambda_{\mathrm{NS}}$. The $\Lambda_{\mathrm{NS}}$ depends on the equation of state (EoS) of NS matter (Shibata \& Taniguchi 2011; Foucart 2012; Kyutoku et al. 2015; Kawaguchi et al. 2015; Foucart et al. 2018). In particular, the optimal conditions leading to the NS tidal disruption and therefore the release of ejecta powering the EM counterpart are the low mass ratio $q=M_{\mathrm{BH}} / M_{\mathrm{NS}}$, large $\chi_{\mathrm{BH}}$, and large $\Lambda_{\mathrm{NS}}$, or, equivalently,

\footnotetext{
3 Hereafter, $\chi_{\mathrm{BH}}=c J / G M_{\mathrm{BH}}^{2}$ is the dimensionless spin parameter and $J$ is the $\mathrm{BH}$ angular momentum.
} 
a stiff EoS (Bildsten \& Cutler 1992; Shibata et al. 2009; Foucart et al. 2013a,b; Kawaguchi et al. 2015; Pannarale et al. 2015a,b; Hinderer et al. 2016; Kumar et al. 2017; Barbieri et al. 2019a). To leading order, the orbital evolution of a compact binary is governed by a combination of the two individual masses, known as chirp mass:

$$
M_{\mathrm{c}}=\frac{\left(M_{1} M_{2}\right)^{3 / 5}}{\left(M_{1}+M_{2}\right)^{1 / 5}} .
$$

Barbieri et al. (2019b) pointed out that systems with chirp masses in the range $1.2 M_{\odot} \lesssim M_{\mathrm{c}} \lesssim 2 M_{\odot}$, depending on the EoS, host a larger variety of configurations. They can either be NSNS or BHNS binaries ${ }^{4}$, and their nature cannot be distinguished through the GW signal analysis alone, at least in low latency (Mandel et al. 2015). We defined these systems as 'ambiguous'. In this range, BHNS and NSNS binaries have different effective tidal deformabilities, and only statistical inference analysis over a large number of detections can provide information on these ambiguous systems from GW data (Farr et al. 2011).

Hinderer et al. (2019) first presented a direct comparison of GW and EM observables from BHNS and NSNS mergers for selected mass ratios. Kawaguchi et al. (2020) showed that BHNS mergers can produce kilonova $(\mathrm{KN})$ emission as bright as the GW170817 case in the optical band and even brighter in the infrared (1-2 mag). They investigated the imprint of the different ejecta properties on the peak time and brightness, suggesting that multi-wavelength $\mathrm{KN}$ observation can unveil the nature of the binary. However, Hinderer et al. (2019) considered only NSNS and BHNS systems involving low-mass NSs $\left(M_{\mathrm{NS}}=1.2 M_{\odot}\right.$ and $\left.1.44 M_{\odot}\right)$ with mass ratio $q=1$ and $q=1.2$, while Kawaguchi et al. (2020) considered BHNS systems with NS mass $M_{\mathrm{NS}}=1.35 M_{\odot}$ and $q=3$ and $q=7$ (simulations presented in Kyutoku et al. 2015), outside the critical interval of ambiguous systems.

In Barbieri et al. (2019b) we showed that the KN emission from ambiguous NSNS and BHNS mergers, corresponding to the same $M_{\mathrm{c}}$, can be very different due to the difference in the properties of their discs and ejecta. NSNS binaries with ambiguous chirp masses host either an NS with $M_{\mathrm{NS}} \sim 1.4$ and a very massive NS ( $\lesssim 2 M_{\odot}$, close to the maximum allowed value, $M_{\mathrm{NS}}^{\max }$ ) or two NSs with $\sim 1.6-1.8 M_{\odot}$. In the latter case, the merger of massive, symmetric, and low- $\Lambda_{\mathrm{NS}}$ stars produces very little ejecta (see Fig. 28 of Radice et al. 2018a and Fig. 2 of Barbieri et al. 2019b) and the KNe from these systems can be very dim. The variety of configurations consistent with the same ambiguous chirp mass gives rise to a potentially broad diversity of possible KN light curves, and in Barbieri et al. (2019b) we showed that BHNS binaries in this range are optimal systems for ejecta production. They can be accompanied by bright and distinguishable $\mathrm{KNe}$ despite degeneracies induced by the large set of physical parameters associated with a given system (see Fig. 4 in Barbieri et al. 2019b). Comparisons between BHNS and NSNS mergers with mass ratio $q \sim 2$ and NSs close to the maximum mass, corresponding to ambiguous systems, are lacking, and this was our motivation to study these systems in more detail in this paper.

Distinguishing the nature of the compact object companion to the NS in these systems is of great value since we can (i) narrow down the uncertainties on the maximum mass of NSs, $M_{\mathrm{NS}}^{\mathrm{max}}$; (ii) discover the existence of $\mathrm{BHs}$ close to the maximum NS mass, and thus the lack of the hypothesised 'lower mass

\footnotetext{
4 In this work we assume that the NS and BH mass distributions are adjacent and form a continuum with no lower mass gap (see the discussion in Sect. 2).
}

gap' between $\sim 2.5 M_{\odot}$ and $\sim 5 M_{\odot}$ between known NSs and BHs. Both results would be of paramount importance to constraining the NS EoS.

The recently discovered GW190425 is an ambiguous binary with $M_{\mathrm{c}}=1.44 \pm 0.02$, for which the presence of a BH (or even two BHs) cannot be completely ruled out (The LIGO Scientific Collaboration \& the Virgo Collaboration 2020; Kyutoku et al. 2020; Han et al. 2020; Tsokaros et al. 2020). The localisation of the source was poor as only a single detector (LIGO Livingston) detected the signal with high $\mathrm{S} / \mathrm{N}$. This prevented any attempts at triangulation using time delays among interferometers.

This work is an application of the study presented in Barbieri et al. (2019b) to the case of GW190425 $5^{5}$, whose chirp mass falls exactly in the ambiguous range. Our analysis employs both the results from the low-latency GW signal properties and the information from the EM follow-up. Moreover, we update our model assuming two physically motivated EoSs and a new fitting formula for the mass of the accretion disc produced in NSNS mergers. Using the information of the chirp mass only, we generate a library of KN light curves for GW190425, with the aim of verifying whether the detection of a transient in the $g, r$, and $J$ bands would have let us distinguish, early in the evolution of the transient, the nature of the compact object companion to the NS, considering the richness of initial configurations compatible with the measured chirp mass and the uncertainties in the EoS.

This work is also motivated by the Neutron Star Interior Composition Explorer (NICER) results (Miller et al. 2019; Riley et al. 2019), which provided complementary indications on the NS EoS from high precision studies of the millisecond pulsar PSR J0030+0451 (Becker et al. 2000) (see Sect. 2). In addition to their potential mass ratio asymmetry, ambiguous systems are expected to modify the mass in the ejecta, and we account for new numerical findings of unequal mass NSNS binaries to calculate the ejecta properties (described in Appendix A). The difference in the expected disc mass causes different masses of ejecta arising as disc outflows, affecting the $\mathrm{KN}$ light curves.

The different system configurations corresponding to the same chirp mass, labelled by the components' masses (and $\mathrm{BH}$ spin for BHNS cases), generate a family of light curves that span a region of the magnitude-time plane. In this work we explore how the light curves from the different configurations are distributed in the magnitude-time domain. We further deepen our analysis considering different sets of model parameters, among them ejecta geometry and grey opacity, to better quantify the level of overlap among the NSNS and BHNS light curves. We also consider BHNS configurations hosting a BH lighter than the maximum NS mass to explore whether light curves from these systems carry any distinctive signature. Moreover, we repeat our analysis on the posterior samples from the GW190425 offline parameter estimation to estimate the level of degeneracy in the light curves and the capability of distinguishing the nature of the merging system between the low-latency and high-latency cases.

Finally, we propose a method to prioritise the follow-up of EM transients with the aim of increasing the chance probability of EM detection using the expected $\mathrm{KN}$ ranges obtained with our model. We apply this method to the low-latency follow-up of GW190425, as it only requires the knowledge of the chirp mass and luminosity distance (both available in low-latency analyses). We note that Margalit \& Metzger (2019) indicated that a rapid release of the chirp mass to the scientific community could help in optimising the EM follow-up. Biscoveanu et al. (2019) also found that the systematics due to low-latency search assumptions

\footnotetext{
5 We stress that, despite the fact that the $\mathrm{BHBH}$ nature cannot be completely ruled out (even if very unlikely), in this work we assume that the GW190425 binary hosted at least one NS.
} 
do not affect the organisation of NSNS candidate EM follow-up when based on the chirp mass.

The paper is organised as follows. In Sect. 2 we discuss whether GW190425 hosts an NSNS or a BHNS merger. In Sect. 3 we estimate the mass loss in NSNS and BHNS binary systems consistent with the chirp mass of GW190425. In Sect. 4 we create a library of light curves and compute peak magnitudes for such systems. In Sect. 4.1 we show how light curves from different configurations are distributed in the magnitude-time domain. In Sect. 4.2 we study the case where BHs have masses below the maximum mass for NSs. In Sect. 4.3 we apply our analysis to the posterior samples from high-latency GW190425 parameter estimation. In Sect. 4.4 we repeat our analysis, adopting the fitting formulae for the mass of the ejecta as recently proposed in Krüger \& Foucart (2020). In Sect. 5 we discuss how knowledge of the chirp mass is critical for the planning of concurrent EM follow-up campaigns. In particular, we apply our argument to the EM follow-ups of GW190425. In Appendix A we describe the newly proposed fitting formula for the disc mass of NSNS mergers. Finally, in Appendix B we study the overlap of the BHNS and NSNS light curves by changing a number of model parameters.

\section{A black hole in GW190425}

The LIGO Scientific Collaboration \& the Virgo Collaboration (2020) reported the detection of the compact object binary merger GW190425, whose chirp mass is $1.44 \pm 0.02 M_{\odot}$. This event is most likely identified as an NSNS merger. The masses of the primary $\left(M_{1}\right)$ and secondary $\left(M_{2}\right)$ star are found to be in the range $1.62 M_{\odot}-1.88 M_{\odot}$ and $1.45 M_{\odot}-1.69 M_{\odot}$ (within the $90 \%$ credible interval), respectively, assuming a low-spin prior $(\chi<0.05)$. Instead, assuming a high-spin prior $(\chi<0.89)$, $M_{1}$ and $M_{2}$ are $1.61 M_{\odot}-2.52 M_{\odot}$ and $1.12 M_{\odot}-1.68 M_{\odot}(90 \%$ credible intervals), respectively. In the case of GW190425, the poorly constrained spins and the uncertainty on the EoS that describes the NS component prevent us from clearly distinguishing an NSNS from a BHNS merger based solely on the GW signal. Therefore, the presence of a BH in GW190425 cannot be excluded (The LIGO Scientific Collaboration \& the Virgo Collaboration 2020; Kyutoku et al. 2020; Han et al. 2020), and this is possible only if stellar BHs exist with masses just above the maximum mass of an NS.

The mass interval from $\sim 2.5 M_{\odot}$ to $\sim 5 M_{\odot}$ is usually defined as the lower mass gap, and as of today EM observations do not show evidence for BHs in this mass interval (Özel et al. 2010, 2012; Farr et al. 2011). The most massive known galactic NS is $\mathrm{J} 0740+6620$, with a measured mass $M=2.14_{-0.09}^{+0.10} M_{\odot}$, while the lightest BHs detected by LVC and observed in Galactic X-ray binaries have masses $7.6_{-2.1}^{+1.3} M_{\odot}$ (The LIGO Scientific Collaboration \& the Virgo Collaboration 2019a) and $7.8 \pm 1.2 M_{\odot}$ (Özel et al. 2010), respectively. However, the core-collapse supernova (SN) explosion models with long explosion timescales and significant fallback presented in Belczynski et al. (2012) and Fryer et al. (2012) can produce remnants with a continuous mass spectrum. Additionally, a recent measurement of a $\mathrm{BH}$ with mass $\sim 3.3_{-0.7}^{+2.8}$ (Thompson et al. 2019), and candidates reported by LVC with at least one component having a mass between $3 M_{\odot}$ and $5 M_{\odot}$ (The LIGO Scientific Collaboration \& the Virgo Collaboration 2019d,e) seem to support the hypothesis of the absence of the lower mass gap.

In Fig. 1 we show the $M_{1}-M_{2}$ configurations compatible with the chirp mass of GW190425 as measured in low latency (The LIGO Scientific Collaboration \& the Virgo Collaboration 2020). The vertical lines in Fig. 1 indicate the maximum NS

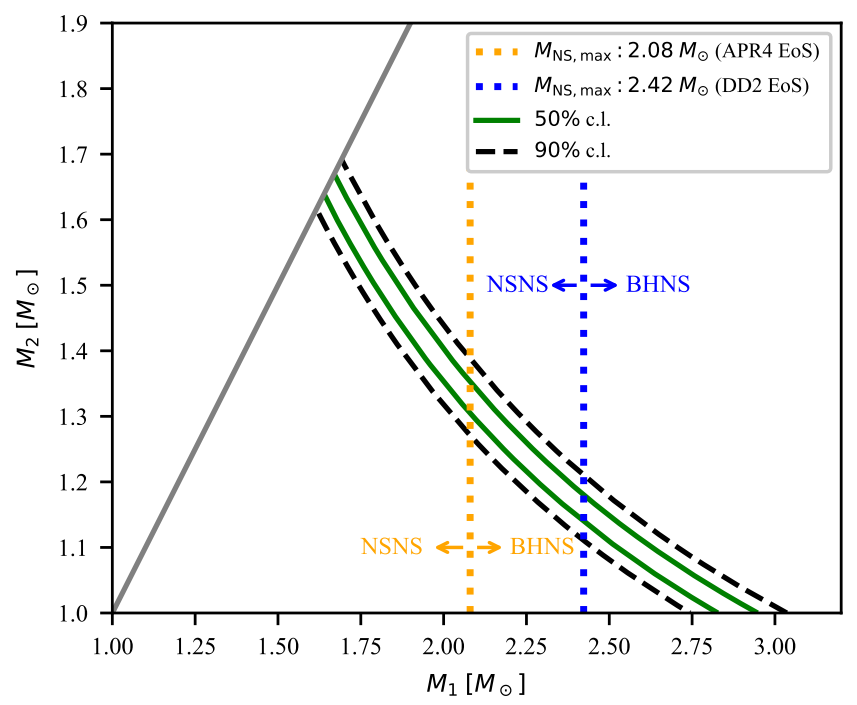

Fig. 1. $M_{1}-M_{2}$ configurations compatible with the inferred value of the chirp mass for GW190425, $M_{\mathrm{c}}=1.44 \pm 0.02 M_{\odot}$. We show the $50 \%$ and $90 \%$ confidence regions in green and dashed black, respectively. Orange and blue vertical dotted lines indicate the NS maximum mass for the EoSs APR4 and DD2, respectively.

mass for two selected EoSs: APR4 (Akmal et al. 1998; Read et al. 2009) and DD2 (Hempel \& Schaffner-Bielich 2010; Typel et al. 2010). They are, respectively, one of the softest and one of the stiffest of the EoSs consistent with the constraints from GW170817 (The LIGO Scientific Collaboration \& the Virgo Collaboration 2019f, 2020; Kiuchi et al. 2019; Radice et al. 2018b) and the NICER (Miller et al. 2019; Riley et al. 2019) results $^{6}$. The APR4 EoS gives $M_{\mathrm{NS}}^{\max }=2.08 M_{\odot}$, while DD2 gives $M_{\mathrm{NS}}^{\max }=2.42 M_{\odot}$. Configurations on the left of these lines correspond to NSNS binaries, while those on the right are BHNS binaries.

\section{Ejecta from GW190425}

In an NSNS merger, partial tidal disruption in the late inspiral phase and crust impact at the merger produce outflows of neutron-rich and mildly neutron-rich material, respectively. Two components can be identified: the 'dynamical ejecta', which are gravitationally unbound and leave the system, and the 'accretion disc', the gravitationally bound component around the merger remnant. Additional outflows can arise from the accretion disc, which we dub 'wind ejecta' (produced by magnetic pressure and neutrino-matter interaction) and 'secular ejecta' (produced by viscous processes, e.g. Dessart et al. 2009; Metzger et al. 2010; Metzger \& Fernández 2014; Perego et al. 2014; Siegel et al. 2014; Just et al. 2015; Siegel \& Metzger 2017; Fujibayashi et al. 2018).

The radioactive decay of elements produced in these ejecta through $r$-process nucleosynthesis powers the $\mathrm{KN}$ emission (Lattimer \& Schramm 1974; Li \& Paczyński 1998; Metzger 2017). In order to calculate the mass $M_{\text {dyn }}$ released in the dynamical ejecta we use the fitting formulae presented in Radice et al. (2018c) (calibrated on a set of high-resolution generalrelativistic hydrodynamic simulations).

As far as the disc mass is concerned, for symmetric mergers Radice et al. (2018c) find that $M_{\text {disc }}$ can be calculated as

6 In Barbieri et al. (2019b) we considered only the SFHo (Steiner et al. 2013) EoS for generating the KN light curves. 

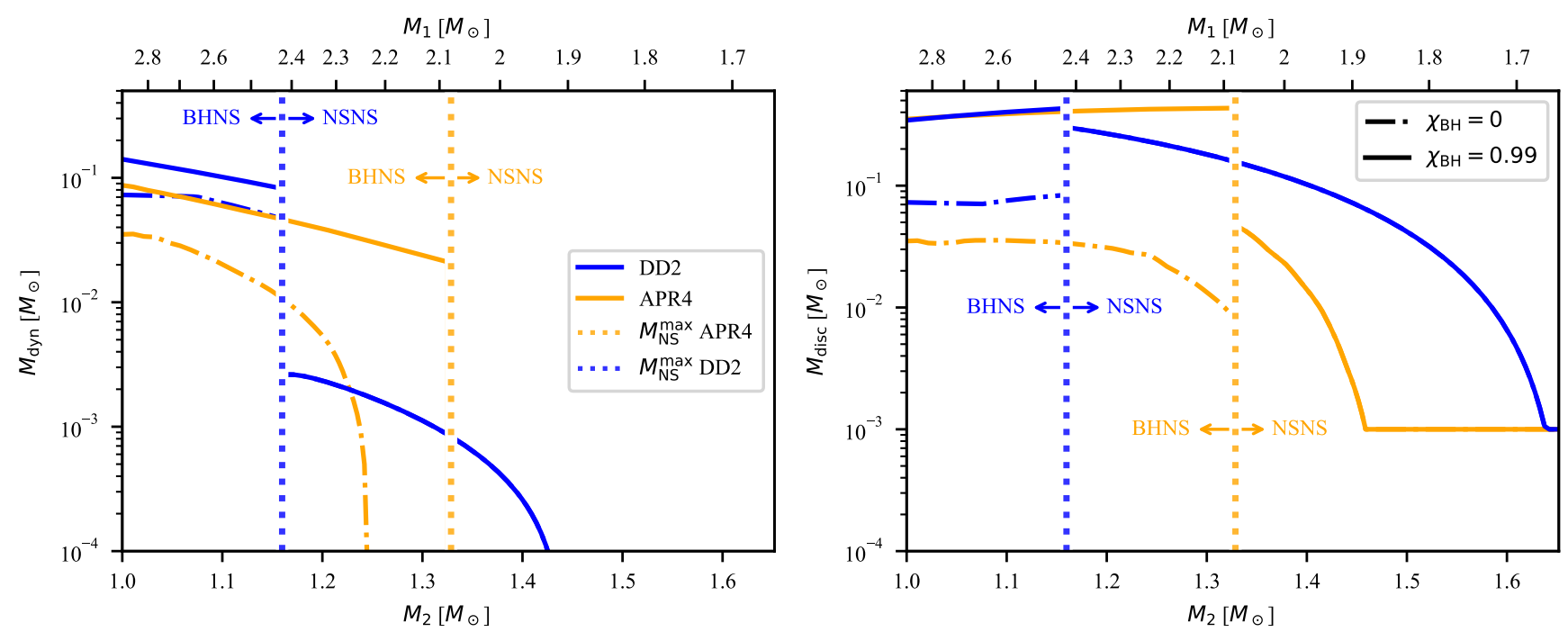

Fig. 2. Dynamical ejecta (left) and accretion disc (right) mass from binary configurations consistent with the chirp mass of GW190425. Orange and blue lines refer to the EoSs APR4 and DD2, respectively. The solid and dot-dashed lines refer to a BH spin of 0.99 and 0, respectively. Dotted vertical lines indicate the maximum NS mass for the two EoSs.

a function of only the binary dimensionless tidal deformability parameter $\tilde{\Lambda}^{7}$. As shown in Fig. 1, we are considering asymmetric NSNS mergers. For these binary configurations, Kiuchi et al. (2019) found that the fitting formula in Radice et al. (2018c) underestimates the accretion disc masses, indicating that $M_{\text {disc }}$ must be calculated as a function of $\tilde{\Lambda}$ and the mass ratio $q$. Thus, to estimate the disc mass we present and adopt a new fitting formula, described in Appendix A (for a further description and application of this formula, see Salafia \& Giacomazzo 2021), that is based on results from the numerical simulations presented in Radice et al. (2018c), Kiuchi et al. (2019), Vincent et al. (2020), and Bernuzzi et al. (2020). This new fitting formula gives values in good agreement with simulations of both symmetric and asymmetric mergers. Therefore, both $M_{\text {dyn }}$ and $M_{\text {disc }}$ depend on the NS masses and tidal deformabilities.

We neglect the possibility of energy injection in the ejecta from a remnant NS state. The NSNS systems considered here carry large total masses and likely collapse promptly (or after a short time) to a $\mathrm{BH}$. We use the recently published fitting formulae by Bauswein et al. (2020) to calculate the threshold mass $M_{\mathrm{thr}}$ for prompt collapse of the binary into a $\mathrm{BH}$. We find that for the EoS APR4 all configurations promptly form a $\mathrm{BH}$, while for DD2 $\sim 64 \%$ of the configurations undergo prompt collapse. As shown by Bernuzzi et al. (2020), though, the prompt collapse in asymmetric binaries does not imply the absence of a disc, as its mass is mainly constituted of bound material from the tidal disruption of the secondary.

Also, BHNS mergers are expected to produce dynamical ejecta and accretion discs if the NS suffers partial tidal disruption before plunging into the BH (Rosswog 2005; Kyutoku et al. 2011; Foucart et al. 2013a). We calculate the dynamical ejecta and accretion disc properties adopting the fitting formulae from Kawaguchi et al. (2016) and Foucart et al. (2018). We follow Barbieri et al. (2019a) to use as fundamental parameters the BH and NS masses, the BH spin and the NS tidal deformability ${ }^{8}$.

\footnotetext{
7 This parameter is defined as $\tilde{\Lambda}=\frac{16}{13} \frac{\left(M_{1}+12 M_{2}\right) M_{1}^{4} \Lambda_{1}+\left(M_{2}+12 M_{1}\right) M_{2}^{4} \Lambda_{2}}{\left(M_{1}+M_{2}\right)^{5}}$ (Raithel et al. 2018).

8 We note that the fitting formula from Kawaguchi et al. (2016) for the mass of dynamical ejecta also depends on $\iota$, which is the angle between the BH spin and the total binary angular momentum. In this work we consider $\iota=0$, corresponding to non-precessing binaries.
}

As discussed in Barbieri et al. (2019a), fixing all the other binary parameters, the larger the $\mathrm{BH}$ spin is, the more ejecta are produced. Therefore, in order to obtain the lower and upper bound on possible ejecta production from GW190425, we assume for the BHNS configurations two spin values: $\chi_{\mathrm{BH}}=0$ and $\chi_{\mathrm{BH}}=0.99$. Figure 2 shows the dynamical ejecta (top) and accretion disc (bottom) masses for configurations consistent with the chirp mass of GW190425.

For the DD2 EoS, BHNS configurations are represented by blue curves on the left of the blue dotted vertical line, which indicates an NS primary with mass equal to its maximum value (i.e., $2.42 M_{\odot}$ ). Similarly for APR4, orange curves on the left of the orange dotted vertical line denote BHNS systems $\left(M_{1}>\right.$ $\left.2.08 M_{\odot}\right)$. Different line styles indicate the different BH spin values. It is clear that BHNS mergers characterised by small mass ratios and low-mass (large- $\Lambda_{\mathrm{NS}}$ ) NSs represent the optimal combination for ejecta production. Indeed in these cases we expect massive dynamical ejecta and discs for both EoSs and both $\mathrm{BH}$ spins. For DD2, BHNS mergers with $\chi_{\mathrm{BH}}=0\left(\chi_{\mathrm{BH}}=0.99\right)$ produce $M_{\text {dyn }} \sim 6-7 \times 10^{-2} M_{\odot}$ and $M_{\text {disc }} \sim 7-8 \times 10^{-2} M_{\odot}$ $\left(M_{\text {dyn }} \sim 10^{-1} M_{\odot}\right.$ and $\left.M_{\text {disc }} \sim 4 \times 10^{-1} M_{\odot}\right)$. For APR4, BHNS mergers with $\chi_{\mathrm{BH}}=0.99$ produce $5 \times 10^{-2} \lesssim M_{\text {dyn }} \lesssim 9 \times 10^{-2} M_{\odot}$ and $M_{\text {disc }} \sim 4 \times 10^{-1} M_{\odot}$. Instead for $\chi_{\mathrm{BH}}=0$ they produce $10^{-2} M_{\odot} \lesssim M_{\text {disc }} \lesssim 3 \times 10^{-2} M_{\odot}$, while dynamical ejecta with $10^{-3} M_{\odot} \lesssim M_{\text {dyn }} \lesssim 3 \times 10^{-2}$ are produced only for $M_{\mathrm{BH}} \gtrsim 2.3 M_{\odot}$.

NSNS configurations are represented by blue curves on the right of the blue dotted vertical line (with $M_{1}<2.42 M_{\odot}$ for the EoS DD2) and orange curves on the right of the orange dotted vertical line (with $M_{1}<2.08 M_{\odot}$ for APR4). These configurations are the worst in producing dynamical ejecta, since massive NSs have small tidal deformability. No dynamical ejecta are produced for APR4, and $M_{\text {dyn }}<3 \times 10^{-3}$ for DD2. Concerning $M_{\text {disc }}$, asymmetric NSNS binaries produce discs with $M_{\text {disc }}<2 \times 10^{-1} M_{\odot}$ for DD2 and $M_{\text {disc }}<5 \times 10^{-2} M_{\odot}$ for APR4. Moving towards symmetric NSNS binaries $(q \rightarrow 1)$, the disc mass significantly decreases.

In the following section we show how the differences in the ejecta properties lead to different $\mathrm{KN}$ luminosities for the BHNS and NSNS case. We caution that the BHNS fitting formulae by Kawaguchi et al. (2016) are calibrated on simulations in the intervals $3 \leq q \leq 7$ and $300 \leq \Lambda_{\mathrm{NS}} \leq 1500$, while 
those by Foucart et al. (2018) in the intervals $1 \leq q \leq 7$ and $280 \leq \Lambda_{\mathrm{NS}} \leq 2070$. The BHNS systems considered in this work fall in the range $1.59 \lesssim q \lesssim 2.9$ and $355 \lesssim \Lambda_{\mathrm{NS}} \lesssim 4180$. NSNS fitting formulae by Radice et al. (2018c) are calibrated on simulations in the ranges $1 \leq q \leq 1.17$ and $125 \leq \tilde{\Lambda} \leq 1440$ and those presented in Appendix A in the ranges $1 \leq q \leq 1.3$ and $125 \leq \tilde{\Lambda} \leq 1440$. The NSNS systems considered in this work fall in the range $1.03 \lesssim q \lesssim 2.09$ and $71 \lesssim \tilde{\Lambda} \lesssim 248$. Thus, ejecta properties for some binary configurations are computed by extrapolating the fitting formulae outside their calibration regimes (see Fig. 3). Therefore, future simulations of mergers in the ambiguous range can lead to modifications of our results. Moreover, during the reviewing process, new fitting formulae for the ejecta mass from NSNS and BHNS mergers were published in Krüger \& Foucart (2020). The authors showed that the fitting formulae by Radice et al. (2018c) and Kawaguchi et al. (2016) give erroneous predictions for very compact NSs (outside their validity range). Despite the fact that the systems considered in this work do not lie in that particular region of the parameter space, for the sake of completeness we carried on the analysis with the new fitting formulae. In Sect. 4.4 we adopt them, showing the analogues of Figs. 2 and 4, and discuss the results.

\section{Kilonova of GW190425}

We compute the KN light curves using the semi-analytical model ${ }^{9}$ presented in Barbieri et al. (2020) (in part based on Grossman et al. 2014; Martin et al. 2015; Perego et al. 2017). This model adopts fitting formulae that provide the mass in the ejecta (presented in Kawaguchi et al. 2016 and Foucart et al. 2018 for BHNS and Radice et al. 2018c and Salafia \& Giacomazzo 2021 for NSNS). In Table 1 we list the assumed model parameters for NSNS mergers (based on Perego et al. 2017) and for BHNS mergers (based on Kawaguchi et al. 2016; Fernández et al. 2017; Just et al. 2015).

In Fig. 4 we show the peak absolute magnitude of $\mathrm{KNe}$ in three relevant bands $(g, r, J)$ from binary configurations consistent with the chirp mass of GW190425. Clearly the KN brightness mirrors the ejecta properties. We find that there is a difference of $\sim 1-1.5 \mathrm{mag}$ at peak between the most luminous $\mathrm{KNe}$ from BHNS and NSNS mergers.

In Fig. 5 we show the KN light curves in all the bands that are associated with binary configurations consistent with the chirp mass $M_{\mathrm{c}}$ of GW190425. For BHNS cases, the lower bounds are obtained considering non-spinning $\mathrm{BHs}\left(\chi_{\mathrm{BH}}=0\right)$, while the upper bounds are obtained considering maximally rotating BHs $\left(\chi_{\mathrm{BH}}=0.99\right)$. For DD2, BHNS KNe are always brighter than the NSNS case in the range of absolute magnitudes considered $[-13,-18]$ (except for the $J$ band, where this holds from $\sim 2$ days to $\sim 12$ days). For APR4, the KN envelope associated with NSNS mergers overlaps with the BHNS one in the lower (low-luminosity) region. However, a large portion of the BHNS parameter space produce brighter KNe compared to the NSNS cases.

In Fig. 5 we also show the limiting magnitude in the GW190425 EM follow-up with the Zwicky Transient Facility (ZTF; Bellm et al. 2019; Coughlin et al. 2019) in the $g$ and $r$ bands,

\footnotetext{
9 We tested our model against GW170817: Multi-wavelength KN light curves obtained with our model using the parameters inferred for this event (The LIGO Scientific Collaboration \& the Virgo Collaboration 2017b; Perego et al. 2017) are consistent with the observations (Villar et al. 2017). Moreover, our light curve peak magnitudes and time behaviour are consistent with Kawaguchi et al. (2020), who derived NSNS-BHNS KN light curves from radiative transfer simulations (including multiple ejecta component effects).
}
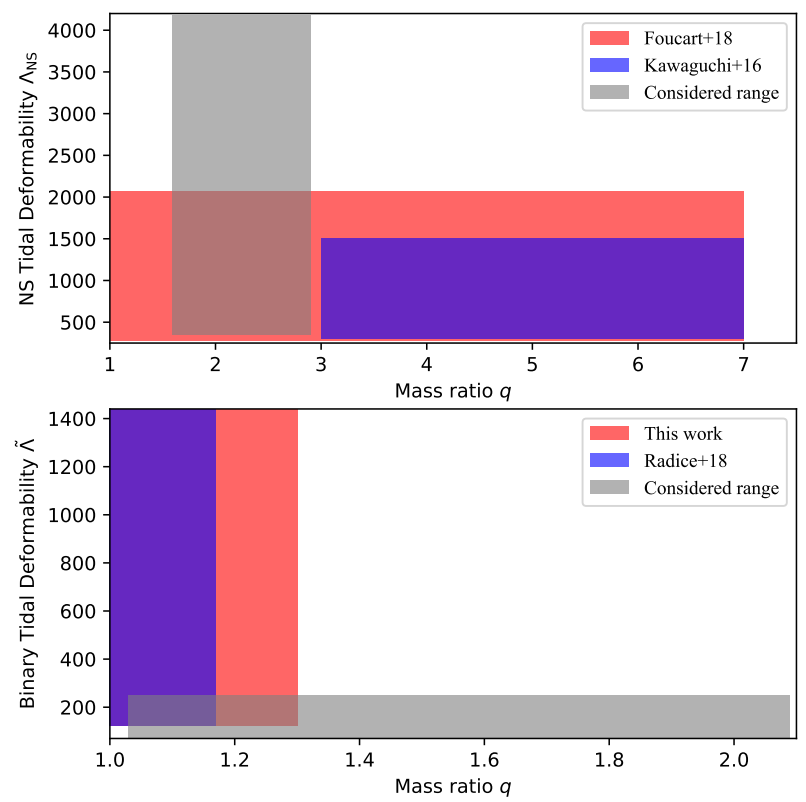

Fig. 3. Parameter ranges where fitting formulae have been calibrated. Top panel: mass ratio and NS tidal deformability ranges on which fitting formulae for BHNS systems by Kawaguchi et al. (2016) (blue) and Foucart et al. (2018) (red) are calibrated, together with the range for BHNS binaries considered in this work (grey), consistent with $M_{\mathrm{c}}=$ $1.44 \pm 0.02 M_{\odot}$. Bottom panel: mass ratio and binary tidal deformability ranges on which disc mass fitting formulae for NSNS systems by Radice et al. (2018c) (blue) and the one presented in the appendix (red) are calibrated, together with the range for NSNS binaries considered in this work (grey), consistent with $M_{\mathrm{c}}=1.44 \pm 0.02 M_{\odot}$.

assuming that the merger happened at a distance $d_{\mathrm{L}}=161 \mathrm{Mpc}$ (The LIGO Scientific Collaboration \& the Virgo Collaboration 2020). We find that BHNS KNe would have been detectable for all (almost all) the binary configurations ${ }^{10}$ for DD2 (APR4) in the first $\sim 4-8$ days. Some NSNS configurations for APR4 (DD2) would have produced detectable $\mathrm{KN}$, with early bright peaks followed by rapid fading (the light curves would have been close to the limiting magnitude after $\sim 3-4$ days).

\subsection{Kilonovae from different binary configurations}

In this section we focus on how the light curves from different binary configurations are distributed in the magnitude-time domain, in order to quantify the degree of superposition of the light curves from BHNS and NSNS binaries shown in Fig. 5.

Assuming flat distributions in $M_{\mathrm{NS}}, M_{\mathrm{BH}}$, and $\chi_{\mathrm{BH}}$ for each EoS, we select some NSNS and BHNS configurations consistent with the chirp mass of GW190425 and show the corresponding KN light curves. The configurations are illustrated in Table 2.

We select equally spaced primary masses and we calculate the corresponding $M_{2}$ using the chirp mass (for NSNS systems with APR4, we start from $M_{1}=1.9 M_{\odot}$ because configurations with a less massive primary produce only very dim emission). For BHNS configurations, we assumed three spin values: $\chi_{\mathrm{BH}}=0,0.5$, and 0.99 .

In the first row of Fig. 6 we show KN light curves for selected NSNS configurations and their expected range. We find

${ }_{10}$ We compared our results with a recent work on the possibility that GW190425 was a BHNS merger (Kyutoku et al. 2020, appeared on arXiv during the writing of this paper). Like us, they find that the $\mathrm{KN}$ associated with a BHNS merger consistent with the chirp mass of GW190425 could have been detected during the EM follow-up. 
Table 1. Assumed ejecta properties for NSNS and BHNS mergers.

\begin{tabular}{cccc}
\hline \hline Parameter & Description & NSNS & BHNS \\
\hline$\xi_{\mathrm{w}}$ & Accretion disc mass fraction flowing in wind ejecta & 0.05 & 0.01 \\
\hline$\xi_{\mathrm{s}}$ & Accretion disc mass fraction flowing in secular ejecta & 0.2 & 0.2 \\
\hline$v_{\mathrm{w}}$ & Wind ejecta velocity & $0.1 \mathrm{c}$ & $0.1 \mathrm{c}$ \\
\hline$v_{\mathrm{s}}$ & Secular ejecta velocity & $0.1 \mathrm{c}$ & $0.1 \mathrm{c}$ \\
\hline$k_{\mathrm{d}}$ & Dynamical ejecta opacity & $15 \mathrm{~cm}^{2} \mathrm{~g}^{-1}$ & $15 \mathrm{~cm}^{2} \mathrm{~g}^{-1}$ \\
\hline$k_{\mathrm{w}}$ & Wind ejecta opacity & $0.5 \mathrm{~cm}^{2} \mathrm{~g}^{-1}$ & $1 \mathrm{~cm}^{2} \mathrm{~g}^{-1}$ \\
\hline$k_{\mathrm{s}}$ & Secular ejecta opacity & $5 \mathrm{~cm}^{2} \mathrm{~g}^{-1}$ & $5 \mathrm{~cm}^{2} \mathrm{~g}^{-1}$ \\
\hline$\theta_{\mathrm{d}}$ & Dynamical ejecta latitudinal opening angle (from the equatorial plane) & $80 \mathrm{deg}^{-1}$ & $17 \mathrm{deg}^{-1}$ \\
\hline$\phi_{\mathrm{d}}$ & Dynamical ejecta azimuthal opening angle & $2 \pi \mathrm{rad}$ & $\pi \mathrm{rad}$ \\
\hline$\theta_{\mathrm{w}}$ & Wind ejecta opening angle (from the polar axis) & $60 \mathrm{deg}$ & $60 \mathrm{deg}$ \\
\hline
\end{tabular}
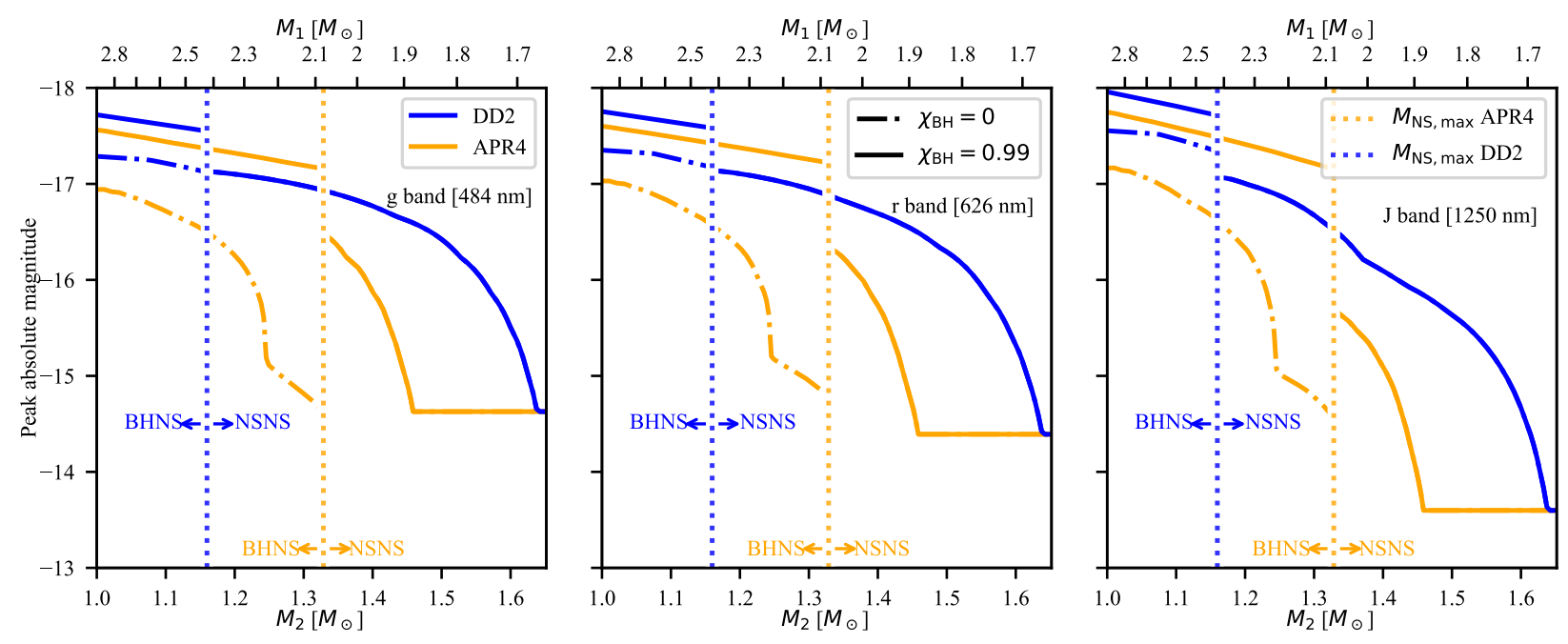

Fig. 4. Peak absolute magnitude of KNe from binary configurations consistent with the chirp mass of GW190425. Left, central, and right panels: respectively, the $g(484 \mathrm{~nm}), r(626 \mathrm{~nm})$, and $J(1250 \mathrm{~nm})$ bands. Orange and blue lines refer to the EoSs APR4 and DD2, respectively. Solid and dot-dashed lines refer to a BH spin of 0.99 and 0, respectively. Dotted vertical lines indicate the maximum NS mass for the two EoSs.

that $\mathrm{KN}$ emission from the different configurations almost uniformly cover the magnitude versus time plane. In the second row of Fig. 6 we show KN light curves for BHNS systems assuming APR4 and the corresponding NSNS KN range from Fig. 5. We find that the majority of BHNS KNe are found in the bright region, while only a few light curves fall in the dim region overlapping with the NSNS KN range (in particular, those corresponding to low spin and small mass ratio). Therefore, the overlap at almost all times between the BHNS and NSNS KN expected ranges for APR4 and presented in Fig. 5 is in reality limited to only a few configurations. The same holds for the late time overlaps for the EoS DD2 (bottom row of Fig. 6). This strengthens the possibility of distinguishing the nature of the ambiguous merging system through the observation of the associated $\mathrm{KN}$.

\subsection{Possible ejecta and kilonova of GW190425 with BH masses below $M_{\mathrm{NS}, \max }$}

In this section we repeat the analysis performed in Sects. 3-5, allowing the BHs to have masses comparable with those of NSs. Therefore, here we adopt a more agnostic approach, describing BHNS binaries without imposing the condition $M_{\mathrm{BH}}^{\min }=$ $M_{\mathrm{NS}}^{\max }$ but simply considering that the primary component is a
$\mathrm{BH}$ and the secondary is an NS. Some studies have demonstrated that such a BHNS system would be compatible with GW170817 multi-messenger observations (Hinderer et al. 2019; Foucart et al. 2019; Coughlin \& Dietrich 2019), although the NSNS nature seems more likely. In this case the ambiguous chirp masses are represented by all the values smaller than the maximum $M_{\mathrm{c}}$ for an NSNS system $\left(\sim 1.81 M_{\odot}\right.$ for APR4 and $\sim 2.11 M_{\odot}$ for DD2).

Figure 7 is analogous to Fig. 2, showing the dynamical ejecta (top) and accretion disc (bottom) masses for configurations consistent with the chirp mass of GW190425. For a given $\chi_{\mathrm{BH}}$, the general trend for the dynamical ejecta is that $M_{\text {dyn }}$ decreases for more symmetric BHNS configurations. Also, $M_{\text {disc }}$ decreases for $q \rightarrow 1$, except for systems with large $\chi_{\mathrm{BH}}$ that always produce massive accretion discs. Obviously the results for NSNS systems and BHNS configurations with $M_{\mathrm{BH}} \geq M_{\mathrm{NS}}^{\mathrm{max}}$ are the same as above. The crucial difference is that now there are BHNS configurations (with low $\chi_{\mathrm{BH}}$ ) producing low-mass ejecta or no ejecta at all. This results in a widening of the expected BHNS $\mathrm{KN}$ range in the lowluminosity region, as shown in Figs. 8 and 9. Therefore, we find that KN light curves from BHNS mergers with low-spin and very low-mass BHs (below $M_{\mathrm{NS}}^{\max }$ ) cannot be distinguished from NSNS case. However, the detection of a KN brighter than the 

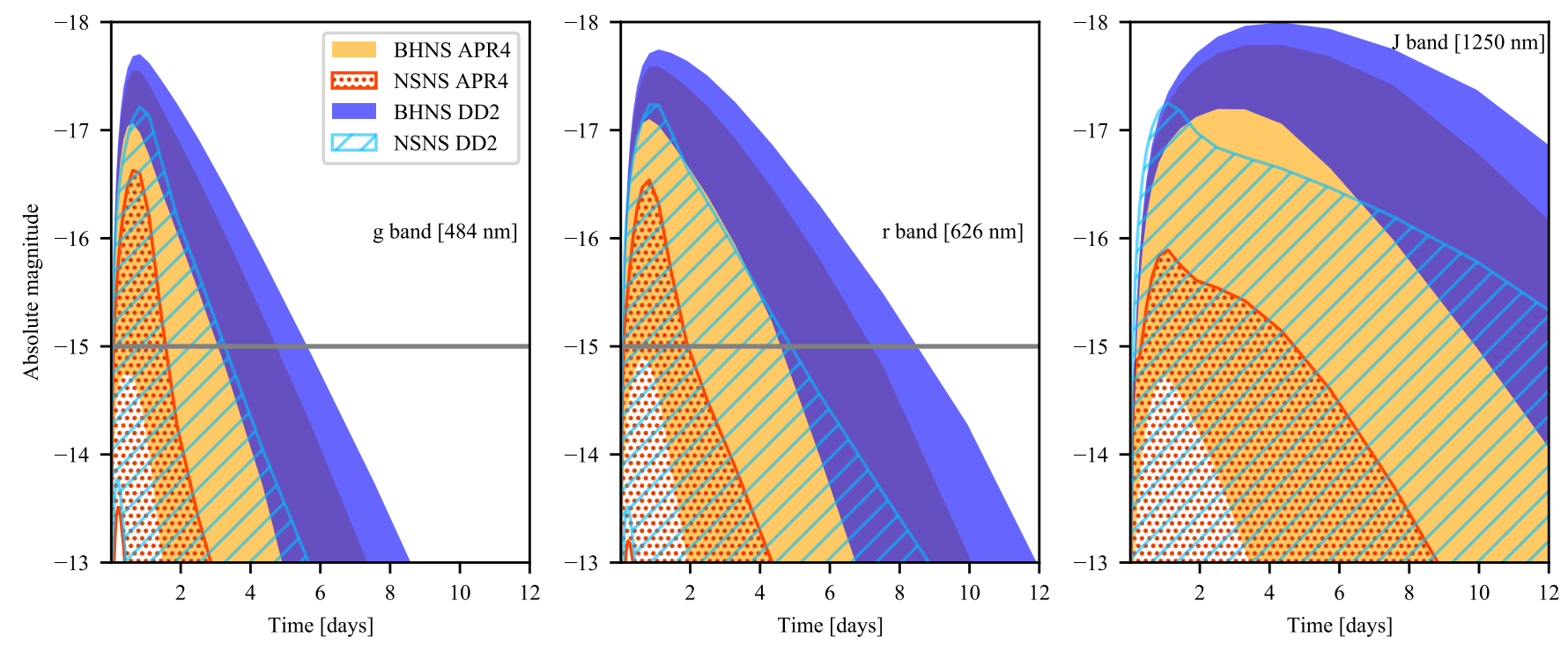

Fig. 5. Range of KN light curves for binary configurations consistent with the chirp mass of GW190425. The ranges are computed including the uncertainties in the fitting formulae for the ejecta and disc masses. For BHNS cases, upper bounds are obtained considering $\chi_{\mathrm{BH}}=0.99$, while lower bounds are obtained considering $\chi_{\mathrm{BH}}=0$. Left, central, and right panels: respectively, the $g(484 \mathrm{~nm}), r(626 \mathrm{~nm})$, and $J(1250 \mathrm{~nm})$ bands. The orange and blue regions refer to BHNS mergers for the EoSs APR4 and DD2, respectively. Dark orange dotted and light blue hatched regions refer to NSNS mergers for the EoSs APR4 and DD2, respectively. Grey horizontal lines correspond to the limiting magnitude in the GW190425 EM follow-up with ZTF, assuming a distance $d_{\mathrm{L}}=161 \mathrm{Mpc}$.

Table 2. Selected NSNS and BHNS configurations compatible with the chirp mass of GW190425, for the analysis in Sect. 4.1.

\begin{tabular}{ccc}
\hline \hline & $M_{1}\left[M_{\odot}\right]$ & $M_{2}\left[M_{\odot}\right]$ \\
\hline \multirow{3}{*}{ NSNS (APR4) } & 1.90 & 1.45 \\
\cline { 2 - 3 } & 1.95 & 1.41 \\
\cline { 2 - 3 } & 2.00 & 1.38 \\
\hline & 1.70 & 1.61 \\
\cline { 2 - 3 } NSNS (DD2) & 1.80 & 1.52 \\
\cline { 2 - 3 } & 1.90 & 1.45 \\
\cline { 2 - 3 } & 2.00 & 1.38 \\
\cline { 2 - 3 } & 2.10 & 1.32 \\
\cline { 2 - 3 } & 2.20 & 1.26 \\
\cline { 2 - 3 } & 2.30 & 1.21 \\
\hline \multirow{3}{*}{ BHNS (APR4) } & 2.40 & 1.17 \\
\cline { 2 - 3 } & 2.20 & 1.26 \\
\cline { 2 - 3 } & 2.40 & 1.17 \\
\cline { 2 - 3 } & 2.60 & 1.09 \\
\hline \multirow{3}{*}{ BHNS (DD2) } & 2.80 & 1.03 \\
\cline { 2 - 3 } & 2.50 & 1.13 \\
\cline { 2 - 3 } & 2.70 & 1.06 \\
\hline
\end{tabular}

allowed NSNS range would still be consistent only with a BHNS merger.

\subsection{Kilonovae associated with GW190425 from GW posterior samples}

In this section we analyse the $\mathrm{KN}$ light curves obtained from the posterior samples of GW signal analysis of GW19042511. In particular, we consider the samples obtained using the 'PhenomDNRT' waveform approximant and the high-spin prior

\footnotetext{
11 Available at https://dcc . ligo.org/LIGO-P2000026/public
}

(The LIGO Scientific Collaboration \& the Virgo Collaboration 2020). We extracted the $M_{1}, M_{2}, \Lambda_{1}, \Lambda_{2}, \tilde{\Lambda}$, and $\chi_{\text {eff }}$ (from which the primary's spin can be obtained as $\left.\chi_{1}=\chi_{\mathrm{eff}}\left(M_{1}+M_{2}\right) / M_{1}\right)$ samples. From these parameters, the ejecta properties could be computed using the fitting formulae indicated in Sect. 3. We then computed the $\mathrm{KN}$ light curves using the model described in Sect. 4.

In the top row of Fig. 10 we show with grey lines the $\mathrm{KN}$ light curves for samples representing BHNS configurations, assuming that $M_{\mathrm{BH}}^{\min }=M_{\mathrm{NS}}^{\max }$. In the central row of Fig. 10 we show with grey lines the KN light curves for all samples assuming that the primary object is a BH. In the bottom row of Fig. 10 we show with grey lines the KN light curves for samples representing NSNS configurations. In each row, blue (resp. orange) lines represent the selected samples consistent with DD2 (resp. APR4). In the top row of Fig. 10 we find that, assuming $M_{\mathrm{BH}}^{\min }=M_{\mathrm{NS}}^{\max }$, the KNe from BHNS systems for different EoS only slightly overlap with those from NSNS systems. Instead, in the central row of Fig. 10 we find a larger overlap (particularly in the low-luminosity region). This is due to the presence of binary configurations producing low-mass ejecta for both EoS (as explained in Sect. 4.2). The dashed lines in the first two rows of Fig. 10 represent the KN ranges for all NSNS configurations (black), those consistent with DD2 (aqua) and those consistent with APR4 (orange). Assuming $M_{\mathrm{BH}}^{\min }=M_{\mathrm{NS}}^{\max }$, we find that for DD2, BHNS KNe are brighter than NSNS ones (at almost all times in the $g$ and $r$-band, after $t \sim 2$ days in the $J$-band). For APR4, the BHNS KNe are brighter than NSNS ones for $t \gtrsim 3$ day in the $g$-band, $t \gtrsim 4$ days in the $r$-band and $t \gtrsim 8$ days in the $J$-band, while at earlier times there are some overlaps. Therefore, we find that degeneracies are still present also considering the high-latency parameter estimation analysis, although reduced with respect to the low-latency estimates. However, the results of the previous analysis are confirmed, as the brighter $\mathrm{KNe}$ would be consistent only with a BHNS merger. 

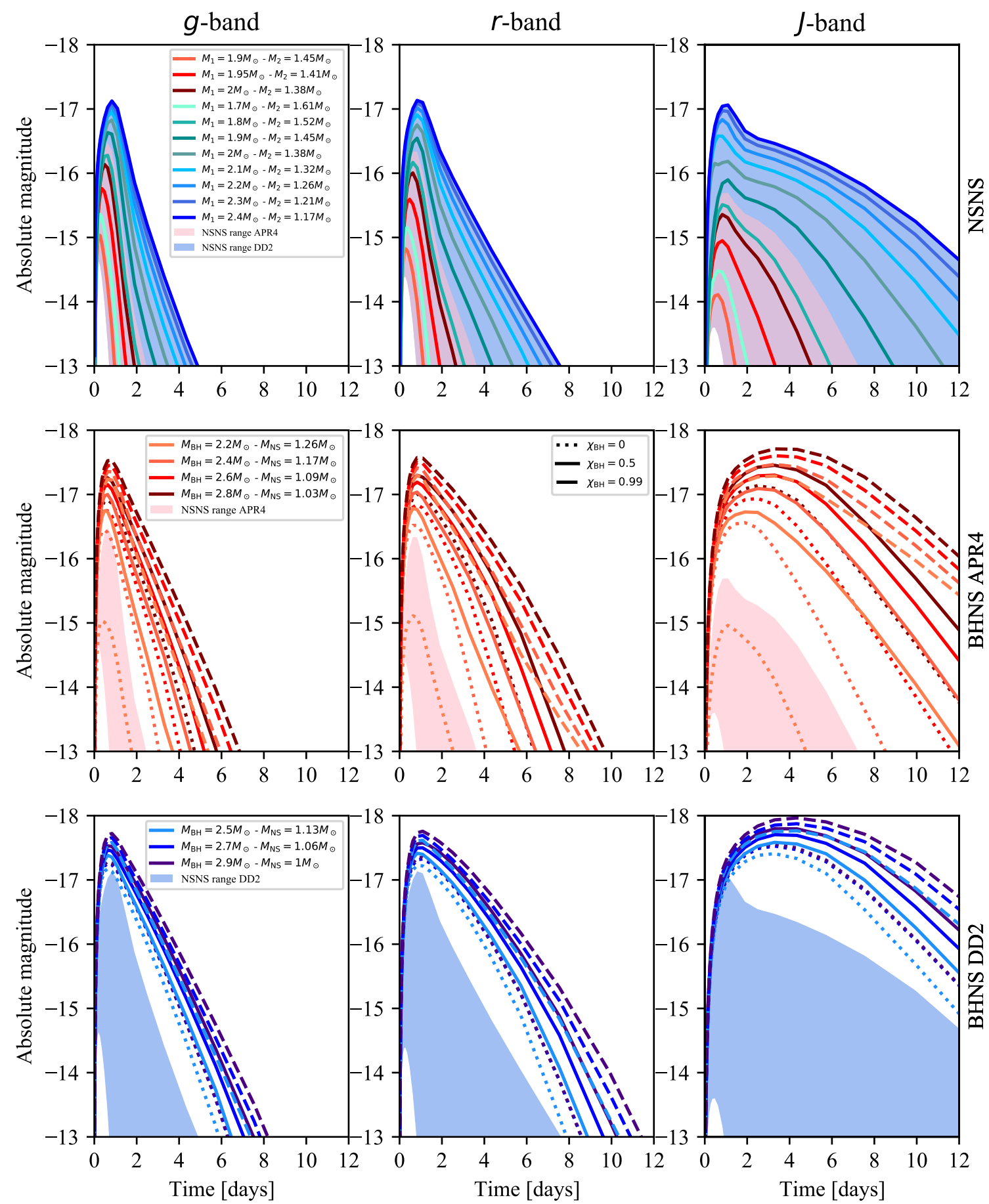

Fig. 6. KN light curves (lines) and expected ranges (filled areas) for different configurations and EoS choices. Top row: NSNS kilonovae for APR4 (red tones) and DD2 (blue tones). Lines show single examples, while the filled areas show the encompassed ranges. Central row: KN light curves from selected BHNS configurations and expected NSNS KN ranges for APR4. Bottom row: same as the central row, but for the DD2 EoS. In each panel the colours indicate different binary component masses (legend in the first column). Line styles indicate different BH spins (legend in the central panel).

If we assume that BHs can have masses below $M_{\mathrm{NS}}^{\max }$, BHNS and NSNS KN ranges overlap because, as already explained, moderate spin - almost equal mass BHNS binary configurations produce low-mass ejecta. We stress that the KN ranges in Fig. 10 are slightly different from those in Fig. 5. This is due to the fact that, in order to select samples consistent with an EoS, we require that $\tilde{\Lambda}^{\mathrm{s}}-0.05 \tilde{\Lambda}^{\mathrm{s}} \leq \tilde{\Lambda}\left(M_{1}^{\mathrm{s}}, M_{2}^{\mathrm{s}}, \operatorname{EoS}\right) \leq \tilde{\Lambda}^{\mathrm{s}}+0.05 \tilde{\Lambda}^{\mathrm{s}}$, where $\tilde{\Lambda}^{\mathrm{s}}, M_{1}^{\mathrm{s}}$, and $M_{2}^{\mathrm{s}}$ are the binary tidal deformability, primary, and secondary mass from the samples, respectively.

\subsection{Repeated analysis assuming the fitting formulae proposed by Krüger \& Foucart (2020)}

As anticipated in Sect. 3, Krüger \& Foucart (2020) found that the fitting formulae by Radice et al. $(2018 \mathrm{c})^{12}$ and

${ }^{12}$ Krüger \& Foucart (2020) actually discuss the NSNS dynamical ejecta fitting formula by Dietrich \& Ujevic (2017), but we notice that the formula by Radice et al. (2018c) has the same form, being just a recalibration on new simulation results. 

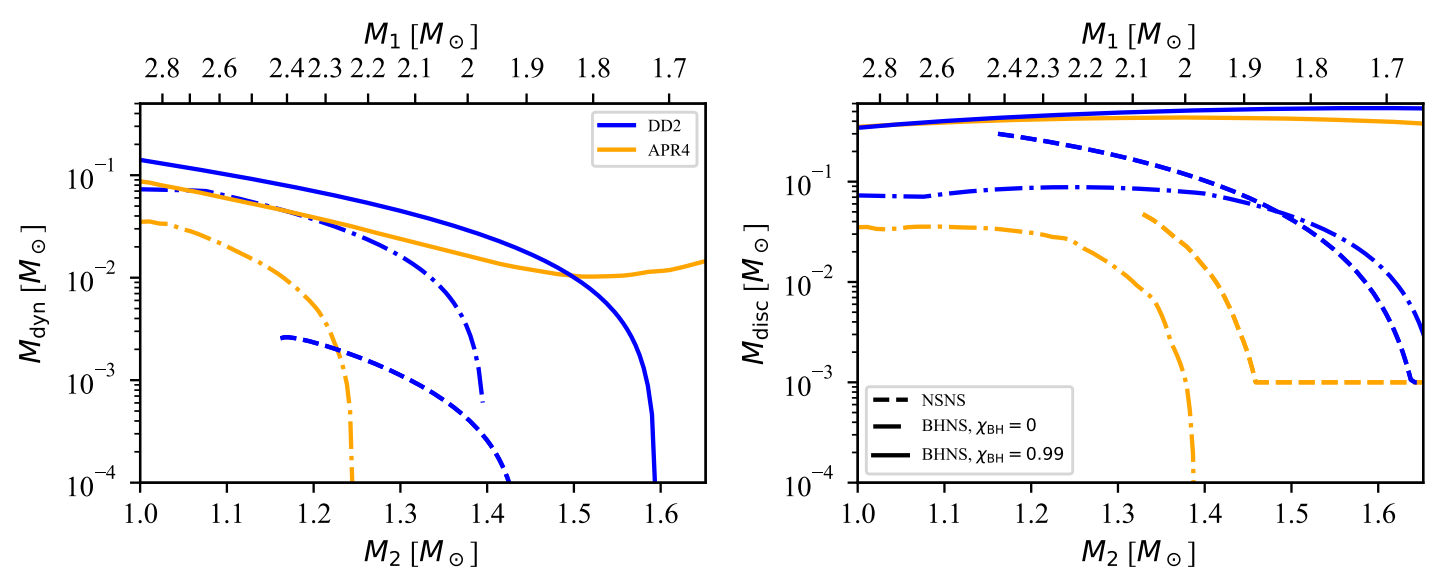

Fig. 7. Same as Fig. 2, but assuming BHs with mass below $M_{\mathrm{NS}}^{\max }$. The solid and dot-dashed lines refer to BHNS systems with $\chi_{\mathrm{BH}}=0.99$ and 0 , respectively, while the dashed line refers to NSNS.
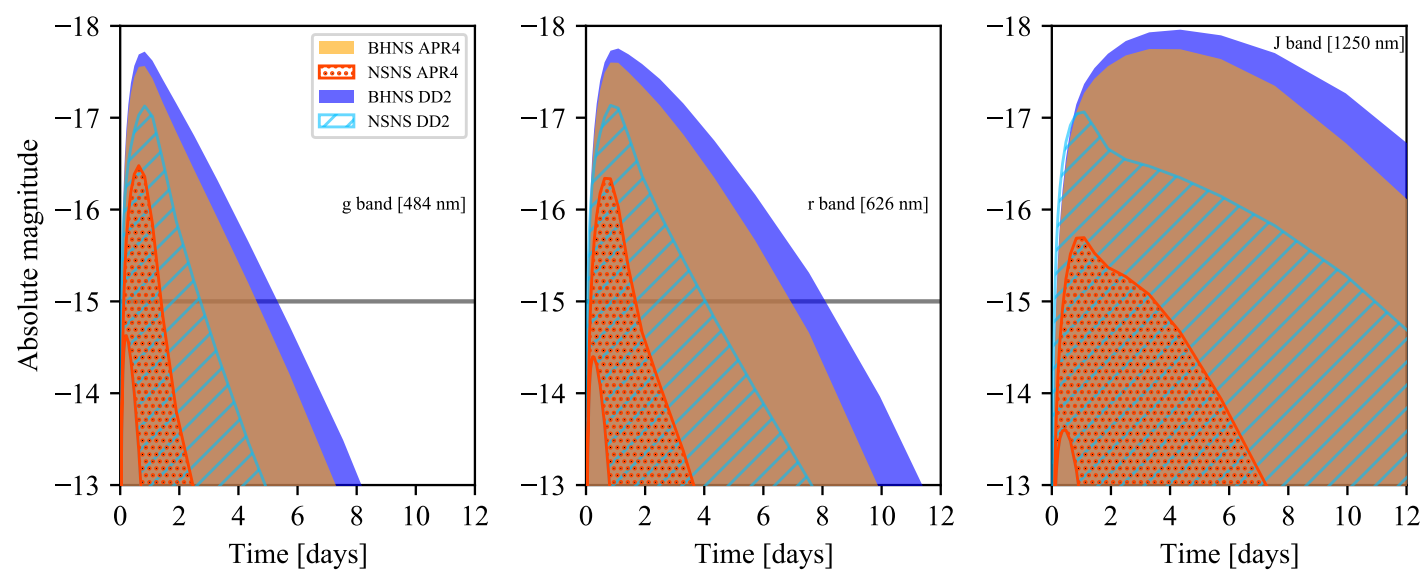

Fig. 8. Same as Fig. 5, but assuming BHs with mass below $M_{\mathrm{NS}}^{\max }$.
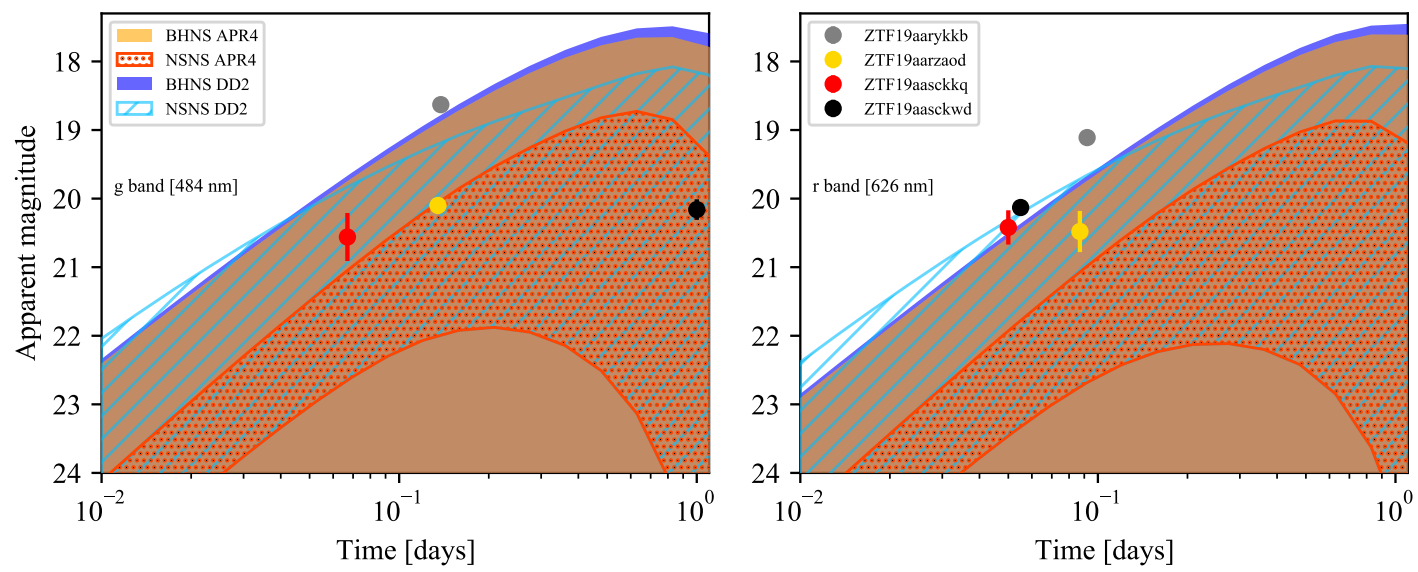

Fig. 9. Same as Fig. 13, but assuming BHs with mass below $M_{\mathrm{NS}}^{\max }$.

Kawaguchi et al. (2016), despite being physically motivated, show an erroneous behaviour for very compact NSs. These formulae predict that mergers involving very massive and compact NSs produce massive ejecta, which is, of course, unexpected (see the right columns of Figs. 3 and 5 of Krüger \& Foucart 2020). Thus, Krüger \& Foucart (2020) propose new fitting formulae inspired by Radice et al. (2018c) and Kawaguchi et al. (2016), and search for a simplified functional form consistent with the largest set of available simulations that also gives expected trends in all the parameter space (see Krüger \& Foucart 2020, Eqs. (4), (6) and (9) and the left columns of Figs. 3 and 5).

The fitting formulae by Krüger \& Foucart 2020 compared to those by Radice et al. (2018c) and Kawaguchi et al. (2016) predict larger masses of NSNS ejecta and smaller ones for BHNS mergers (see Fig. 11). This produces a larger overlap between the two families of light curves, as shown in Fig. 12. By comparing Fig. 12 with Fig. 5 we find that identifying the nature of the merging system through $\mathrm{KN}$ observations is still possible, 

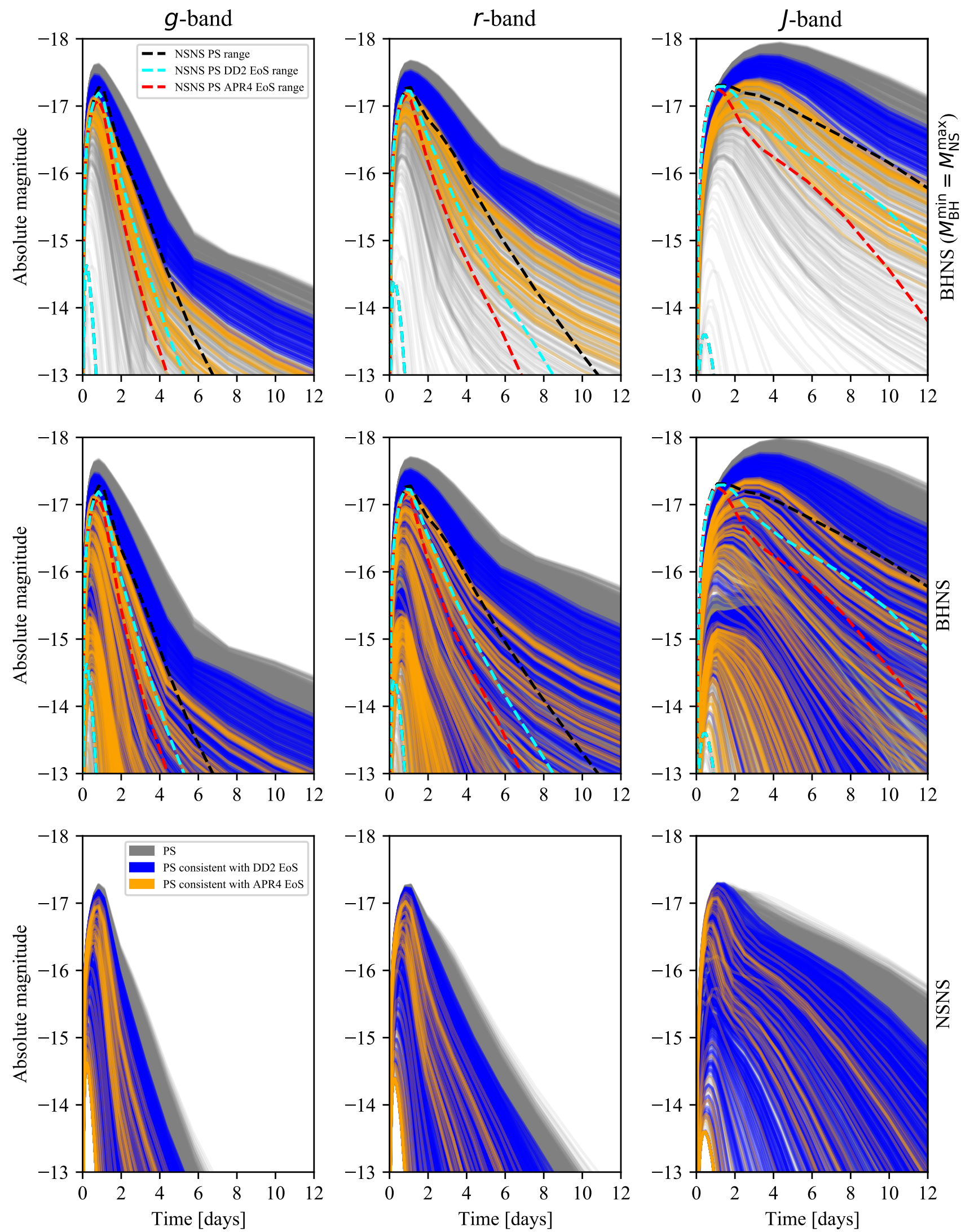

Fig. 10. KN light curves for the GW190425 posterior samples. Top row: samples consistent with BHNS mergers (assuming $M_{\mathrm{BH}}^{\min }=M_{\mathrm{NS}}^{\max }$ ). Central row: all samples, considering that the primary object is a BH. Bottom row: samples consistent with NSNS mergers. Blue and orange lines indicate samples consistent with the EoSs DD2 and APR4, respectively. Dashed black, aqua, and red lines in the first two rows indicate the NSNS KN ranges for, respectively, all EoSs, DD2, and APR4. We consider the $g$-band (left column), $r$-band (central column), and J-band (right column). 

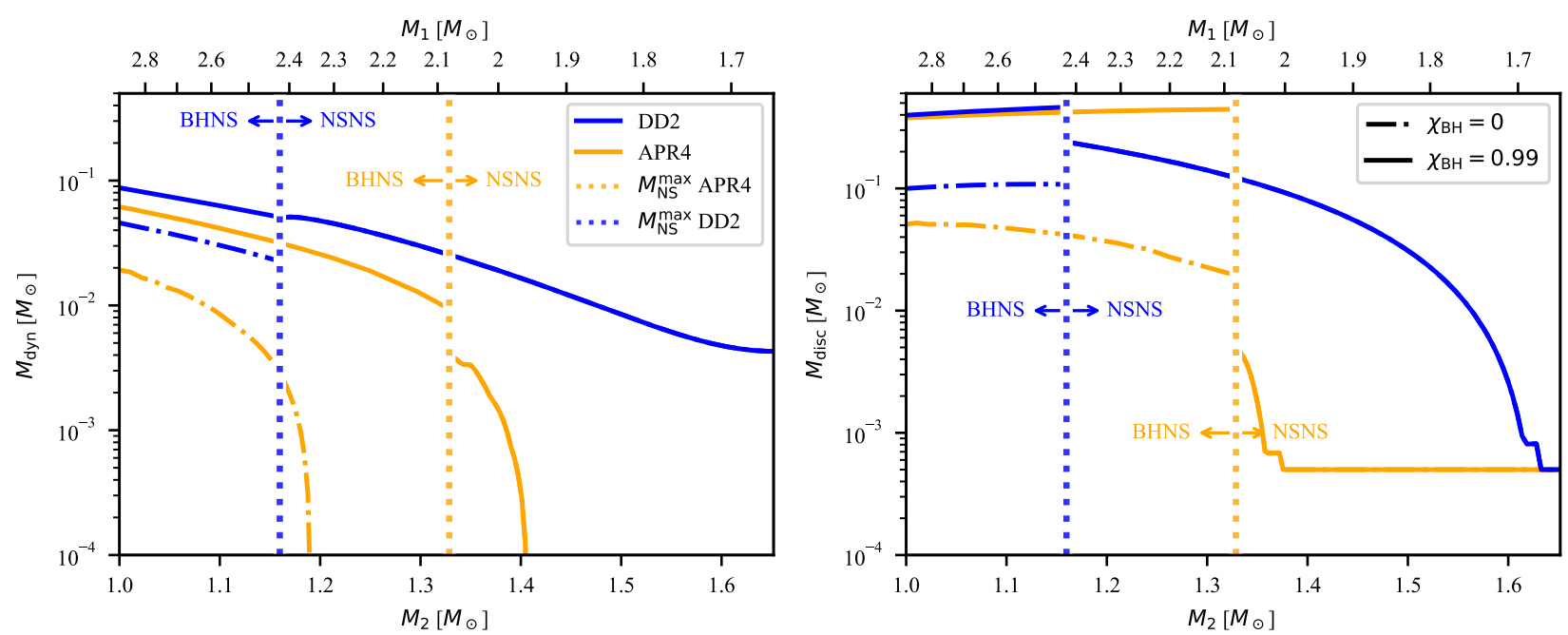

Fig. 11. Same as Fig. 2, but adopting the fitting formulae presented in Krüger \& Foucart (2020).
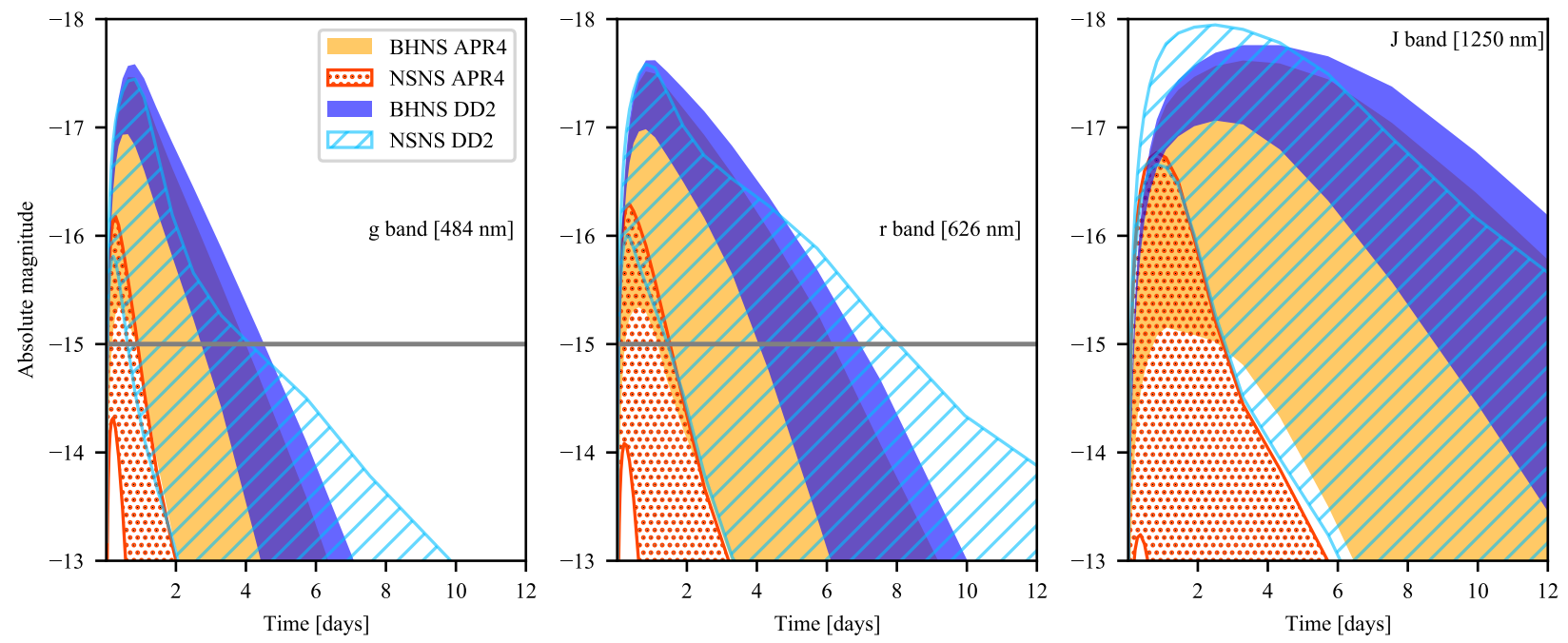

Fig. 12. Same as Fig. 5, but adopting the fitting formulae presented in Krüger \& Foucart (2020).

although with substantial differences compared to our previous analysis. Now for APR4 all the BHNS KN light curves are brighter than the NSNS ones after $\sim 1$ day in the $g$ band, $\sim 2$ days in the $r$ band and $\sim 3$ days in the $J$ band. For DD2, the NSNS and BHNS KN ranges shows a large overlap, with the majority of BHNS KNe (see discussion in Sect. 4.1) being brighter than the NSNS ones for $2 \lesssim t \lesssim 5$ days in the $g$ and $r$ bands and $t \gtrsim 6$ days in the $J$ band. Moreover for DD2 some NSNS KNe are brighter than the BHNS ones at late times ( $t \gtrsim 6$ days) in the $g$ and $r$ bands (although this happens at low luminosities) and at early times ( $t \lesssim 4$ days) in the $J$ band.

In summary, when adopting the fitting formulae of Krüger \& Foucart (2020), the nature of ambiguous mergers can be identified more easily for soft EoSs such as APR, while for stiff EoSs such as DD2 there exists a larger overlap of KN ranges. By contrast, adopting the fitting formulae presented in Radice et al. (2018c) and Kawaguchi et al. (2016), the opposite holds true.

This discussion stresses the crucial need of new NSNS merger numerical simulations, specially in the 'extreme' regions of the parameter space, with largely asymmetric binaries hosting at least one very compact NS, with the aim of finding a valid extension of the present NSNS ejecta fitting formulae for these extreme binaries.

\section{EM follow-up strategy with the knowledge of the chirp mass}

The possibility to distinguish the nature of the merging system for an ambiguous event is related to the detection of the associated KN. This is not a simple achievement, as from the analysis of the GW signal the uncertainties on the localisation volume (obtained by combining the sky localisation and the distance estimates) can be very large. Thousands of bright galaxies (and many more transients) could be present in this volume, making the identification of the $\mathrm{KN}$ associated with the merger very challenging. In the best scenario the $\mathrm{KN}$ is identified after some time, and the short-lived, rapidly decaying transients are lost. In the worst scenario, the $\mathrm{KN}$ is never identified and all the EM counterparts are lost.

In Fig. 5 we show that, knowing the chirp mass, we can calculate the expected KN light curves ranges. This could provide useful criteria to optimise the EM follow-up strategy. Indeed the observation of transients consistent with KN emission at their first detection could be prioritised for the subsequent photometric and/or spectroscopic follow-up, aimed at classifying them. This could enhance the probability of discovering the EM counterpart to the GW event. 

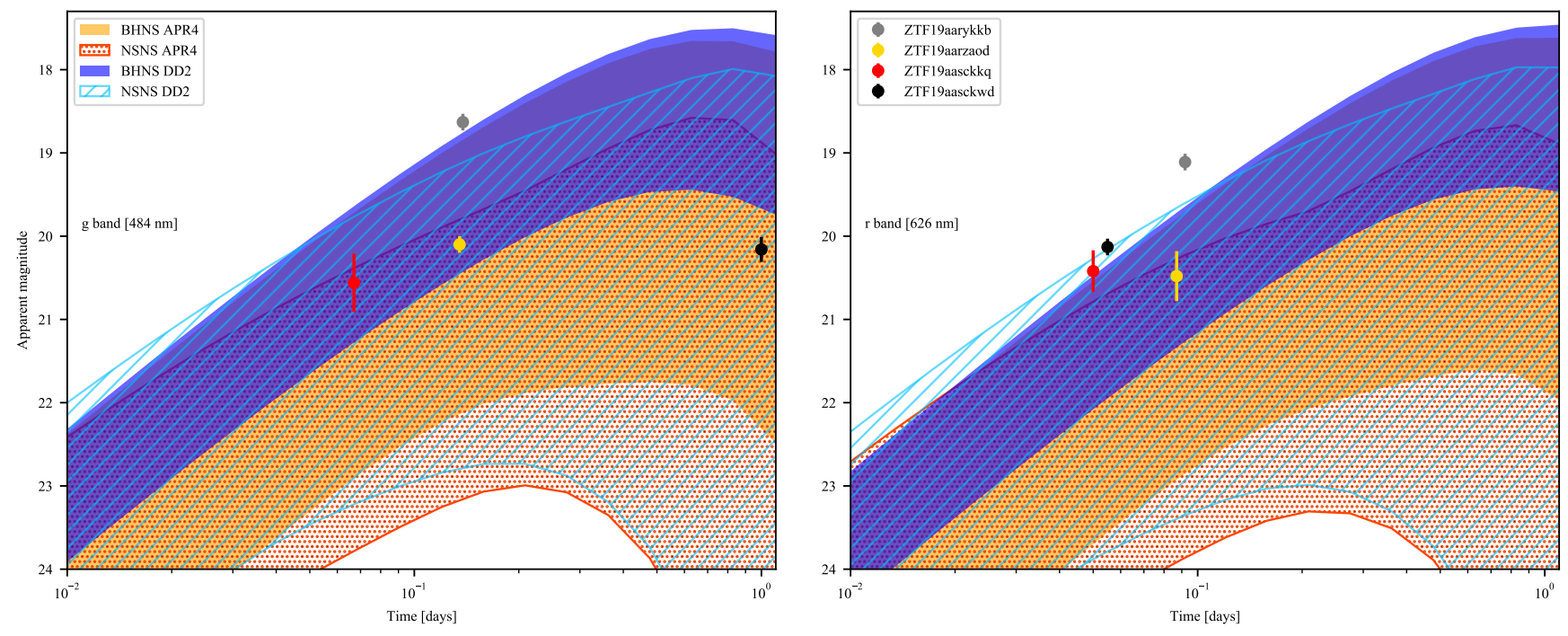

Fig. 13. Range of KN light curves for binary configurations consistent with the chirp mass of GW190425. The ranges are computed including the uncertainties in the fitting formulae for the ejecta and disc masses. Upper bounds are obtained considering $d_{\mathrm{L}}=110 \mathrm{Mpc}\left(\right.$ and $\chi_{\mathrm{BH}}=0.99$ for BHNS cases), while lower bounds are obtained considering $d_{\mathrm{L}}=200 \mathrm{Mpc}$ (and $\chi_{\mathrm{BH}}=0$ for BHNS cases). Coloured points with error bars are the first detections by ZTF of promising candidate EM counterparts to the event. Left and right panels: respectively, the $g(484 \mathrm{~nm})$ and $r(626 \mathrm{~nm})$ bands. Orange and blue regions refer to BHNS mergers for the EoSs APR4 and DD2, respectively. The dark orange dotted region and the light blue hatched region refer to NSNS mergers for the EoSs APR4 and DD2, respectively.

GW190425 was a single interferometer detection. This is one of the reasons why the sky localisation was poorly informative, the $90 \%$ credible sky area being $\sim 8300 \mathrm{deg}^{2}$ (The LIGO Scientific Collaboration \& the Virgo Collaboration $2020)^{13}$. Nonetheless, it is remarkable that the Global Relay of Observatories Watching Transients Happen network observed $\sim 21 \%$ of the sky map (Coughlin et al. 2019). Among all the transients detected during the first $48 \mathrm{~h}, 15$ candidates were particularly interesting (Kasliwal et al. 2019; Anand et al. 2019). After being observed for days they were all classified as $\mathrm{SNe}$ (Coughlin et al. 2019).

In Fig. 13 we show how our argument could be applied to the GW190425 EM follow-up campaign. We calculate the expected apparent magnitude range of $\mathrm{KN}$ light curves using the knowledge of the chirp mass $M_{\mathrm{c}}=1.44 \pm 0.02 M_{\odot}$ and the luminosity distance estimate initially circulated by LVC (The LIGO Scientific Collaboration \& the Virgo Collaboration 2019g) $d_{\mathrm{L}}=155 \pm 45 \mathrm{Mpc}$. Considering APR4 or DD2 as reference EoS to describe NS matter, for each EoS the lower bound is calculated assuming $\chi_{\mathrm{BH}}=0$ and $d_{\mathrm{L}}=200 \mathrm{Mpc}$, while the upper bound assuming $\chi_{\mathrm{BH}}=0.99$ and $d_{\mathrm{L}}=110 \mathrm{Mpc}$. In Fig. 13 we also show the first detections of 4 promising candidates identified by ZTF. These transients were observed for 1-4 days (see Fig. 3 in Coughlin et al. 2019) before being classified as SNe. The first detection of the transients ZTF19aarzaod and ZTF19aasckkq is consistent with the expected $\mathrm{KN}$ ranges, thus subsequent observations would have been anyway needed to understand their nature. Instead the transients ZTF19aarykkb and ZTF19aasckwd are inconsistent with the expected $\mathrm{KN}$ ranges. Therefore, other candidates (consistent with the expected range at the moment of their first detection) could have been observed with higher priority.

We are quite confident in defining ZTF19aarykkb and ZTF19aasckwd as inconsistent to be the GW190425 counterpart. Indeed, these transients would be brighter than the $\mathrm{KN}$ produced

\footnotetext{
13 As a comparison, the GW170817 90\% credible sky area from the low-latency analysis was $\sim 28 \mathrm{deg}^{2}$.
}

by a merger whose chirp mass is the one inferred for GW190425, which happened at the lower bound of the luminosity distance $1 \sigma$ interval, where the $\mathrm{BH}$ is maximally rotating and the NS EoS is one of the stiffest (DD2) among those consistent with GW170817 event.

Figure 14 is obtained adopting the fitting formulae presented in Krüger \& Foucart (2020) and it is the analogous of Fig. 13. In this case we find that only one transient (ZTF19aarykkb) was inconsistent with the expected range of $\mathrm{KN}$ emission at its first detection. While this does not per se question the validity in principle of our proposed approach, it gives a sense of the systematic error in the analysis due to the uncertain fitting formulae, calling for more simulations in the unexplored part of the parameter space.

\section{Summary and results}

In this work we carried out a low-latency analysis based only on the estimates of the GW190425 system's chirp mass and luminosity distance (available a few minutes after the trigger). Such an analysis helps in the planning of EM multi-frequency follow-up campaigns, prioritising the observation of transients to enhance the probability of detecting the EM counterpart. We applied this method to the GW190425 case, constructing NSNS and BHNS KN light curve models for that event, considering two EoSs consistent with current constraints from the signals of GW170817 and the NICER results (including BH spin effects), and assuming a new formula for the mass of the ejecta. We found that if our method had been applied to low-latency follow-up of GW190425, two transients (which were observed for $\sim 24 \mathrm{~h}$ before being discarded) would have been immediately discarded (see Sect. 5).

In Sect. 4 we showed that if one component of GW190425 were a $\mathrm{BH}$, the merger could have produced a far more luminous $\mathrm{KN}$ compared to the NSNS case (examples of KN light curves from BHNS mergers as bright as or brighter than NSNS mergers have already been proposed in e.g., Kawaguchi et al. 2020 and Barbieri et al. 2020). We further found that $\mathrm{KN}$ light curves from 

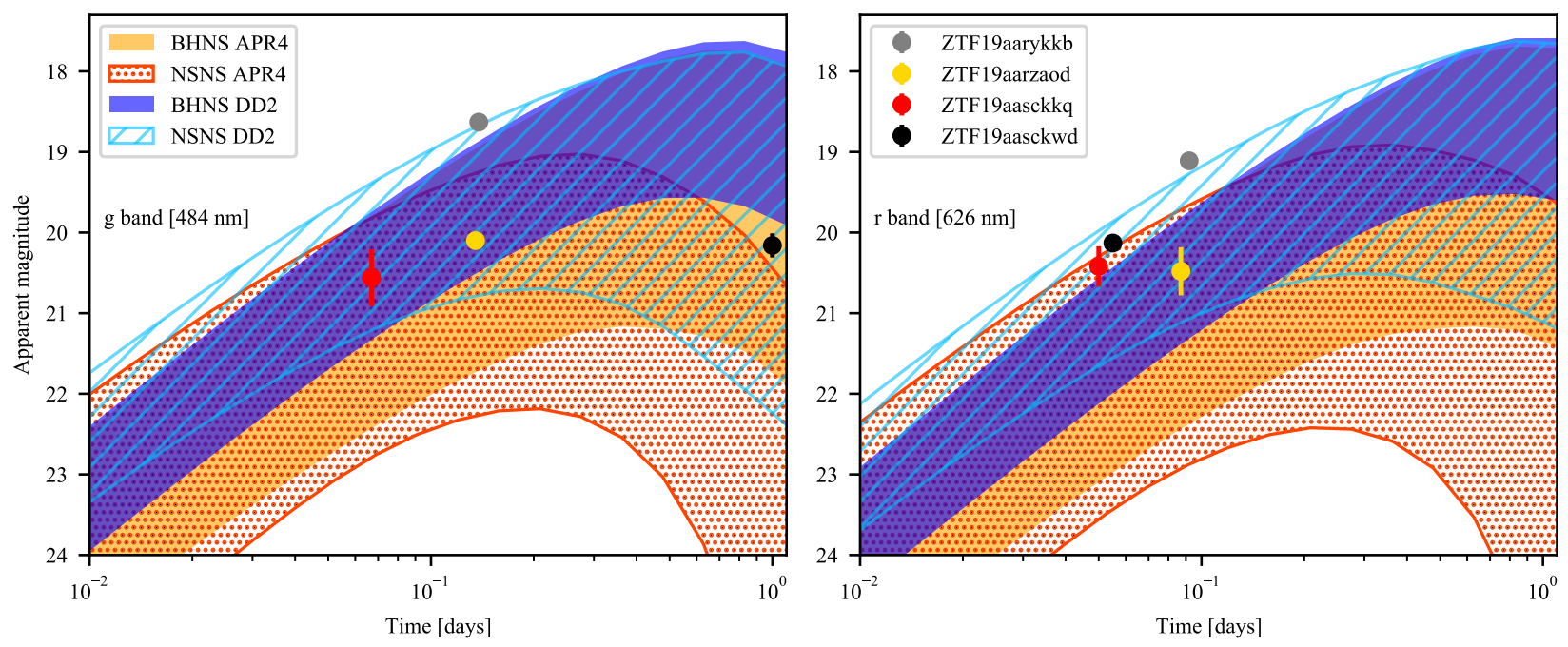

Fig. 14. Same as Fig. 13, but adopting the fitting formulae presented in Krüger \& Foucart (2020).

different NSNS configurations are almost uniformly distributed in the magnitude-time domain, while those from different BHNS configurations are more concentrated in the bright region. The overlap presented in Fig. 4 is thus limited to a few configurations, strengthening our result. Therefore, the putative observation of KN emission associated with GW190425 could have unveiled the nature of the companion to the NS (as suggested in Barbieri et al. 2019b).

In Sects. 4.1, B, and 4.2 we tested the robustness of our results against our model assumptions. Concerning degeneracy, we repeated our analysis on the posterior samples of GW190425 from the high-latency parameter estimation, finding that degeneracy between NSNS and BHNS KN light curves is still present, but reduced. Interestingly, the capability to distinguish the nature of the system using low-latency analysis is comparable to that of the high-latency case. In Sect. 4.2 we further found that if BHs with masses below the maximum mass of NSs exist, the $\mathrm{KNe}$ from such 'very light' BHNS systems can be distinguished from the NSNS case only if the BH spin is large.

Finally, we remark that the identification of a BHNS merger with ambiguous chirp mass would provide the first hint of the existence of 'light' BHs, confuting the presence of a lower mass gap between NS and BH mass distributions. Such a discovery would have an important impact on SN explosion models, favouring those producing a continuous remnant mass spectrum. It would also be crucial for constraining the maximum mass of non-rotating NSs.

Acknowledgements. We thank F. Zappa and S. Bernuzzi for sharing EoS tables. The authors acknowledge support from INFN, under the Virgo-Prometeo initiative. O. S. acknowledges the Italian Ministry for University and Research (MIUR) for funding through project grant 1.05.06.13. M. C. acknowledges the COST Action CA16104 "GWverse", supported by COST (European Cooperation in Science and Technology). During drafting of this paper, M. C. acknowledges kind hospitality by the Kavli Institute for Theoretical Physics at Santa Barbara, under the program "The New Era of Gravitational-Wave Physics and Astrophysics".

\section{References}

Abbott, B. P., Abbott, R., Abbott, T. D., et al. 2017, ApJ, 848, L12 Abbott, R., Abbott, T. D., Abraham, S., et al. 2020, ApJ, 896, L44 Akmal, A., Pandharipande, V. R., \& Ravenhall, D. G. 1998, Phys. Rev. C, 58, 1804

Anand, S., Kasliwal, M. M., Coughlin, M. W., et al. 2019, Gamma-ray Coordinates Network Circulars, 24311
Barbieri, C., Salafia, O. S., Perego, A., Colpi, M., \& Ghirlanda, G. 2019a, A\&A, 625, A152

Barbieri, C., Salafia, O. S., Colpi, M., et al. 2019b, ApJ, 887, L35

Barbieri, C., Salafia, O. S., Perego, A., Colpi, M., \& Ghirlanda, G. 2020, Eur. Phys. J. A, 56, 8

Bauswein, A., Blacker, S., Vijayan, V., et al. 2020, Phys. Rev. Lett., 125, 141103 Becker, W., Trümper, J., Lommen, A. N., \& Backer, D. C. 2000, ApJ, 545, 1015 Belczynski, K., Wiktorowicz, G., Fryer, C. L., Holz, D. E., \& Kalogera, V. 2012, ApJ, 757, 91

Bellm, E. C., Kulkarni, S. R., Graham, M. J., et al. 2019, PASP, 131, 018002

Bernuzzi, S., Breschi, M., Daszuta, B., et al. 2020, MNRAS, 497, 1488

Bildsten, L., \& Cutler, C. 1992, ApJ, 400, 175

Biscoveanu, S., Vitale, S., \& Haster, C.-J. 2019, ApJ, 884, L32

Coughlin, M. W., \& Dietrich, T. 2019, Phys. Rev. D, 100, 043011

Coughlin, M. W., Ahumada, T., Anand, S., et al. 2019, ApJ, 885, L19

Coughlin, M. W., Dietrich, T., Antier, S., et al. 2020, MNRAS, 497, 1181

Dessart, L., Ott, C. D., Burrows, A., Rosswog, S., \& Livne, E. 2009, ApJ, 690, 1681

Dietrich, T., \& Ujevic, M. 2017, Classical Quantum Gravity, 34, 105014

Farr, W., Sravan, N., Cantrell, A., et al. 2011, APS April Meeting Abstracts , 2011, H11.002

Fernández, R., Foucart, F., Kasen, D., et al. 2017, Classical Quantum Gravity, 34, 154001

Foley, R. J., Coulter, D. A., Kilpatrick, C. D., et al. 2020, MNRAS, 494, 190

Foucart, F. 2012, Phys. Rev. D, 86, 124007

Foucart, F., Deaton, M. B., Duez, M. D., et al. 2013a, Phys. Rev. D, 87, 084006

Foucart, F., Buchman, L., Duez, M. D., et al. 2013b, Phys. Rev. D, 88, 064017

Foucart, F., Hinderer, T., \& Nissanke, S. 2018, Phys. Rev. D, 98, 081501

Foucart, F., Duez, M. D., Kidder, L. E., et al. 2019, Phys. Rev. D, 99, 103025

Fryer, C. L., Belczynski, K., Wiktorowicz, G., et al. 2012, ApJ, 749, 91

Fujibayashi, S., Kiuchi, K., Nishimura, N., Sekiguchi, Y., \& Shibata, M. 2018, ApJ, 860, 64

Grossman, D., Korobkin, O., Rosswog, S., \& Piran, T. 2014, MNRAS, 439, 757

Han, M.-Z., Tang, S.-P., Hu, Y.-M., et al. 2020, ApJ, 891, L5

Hempel, M., \& Schaffner-Bielich, J. 2010, Nucl. Phys. A, 837, 210

Hinderer, T., Taracchini, A., Foucart, F., et al. 2016, Phys. Rev. Lett., 116, 063021

Hinderer, T., Nissanke, S., Foucart, F., et al. 2019, Phys. Rev. D, 100, 181101

Just, O., Bauswein, A., Ardevol Pulpillo, R., Goriely, S., \& Janka, H. T. 2015, MNRAS, 448, 541

Kasliwal, M. M., Coughlin, M. W., Bellm, E. C., et al. 2019, Gamma-ray Coordinates Network Circulars, 24191

Kawaguchi, K., Kyutoku, K., Nakano, H., et al. 2015, Phys. Rev. D, 92, 024014

Kawaguchi, K., Kyutoku, K., Shibata, M., \& Tanaka, M. 2016, ApJ, 825, 52

Kawaguchi, K., Shibata, M., \& Tanaka, M. 2020, ApJ, 889, 171

Kiuchi, K., Kyutoku, K., Shibata, M., \& Taniguchi, K. 2019, ApJ, 876, L31

Krüger, C. J., \& Foucart, F. 2020, Phys. Rev. D, 101, 103002

Kumar, P., Pürrer, M., \& Pfeiffer, H. P. 2017, Phys. Rev. D, 95, 044039

Kyutoku, K., Okawa, H., Shibata, M., \& Taniguchi, K. 2011, Phys. Rev. D, 84, 064018

Kyutoku, K., Ioka, K., Okawa, H., Shibata, M., \& Taniguchi, K. 2015, Phys. Rev. D, 92, 044028

Kyutoku, K., Fujibayashi, S., Hayashi, K., et al. 2020, ApJ, 890, L4

Lattimer, J. M., \& Schramm, D. N. 1974, ApJ, 192, L145 
Li, L.-X., \& Paczyński, B. 1998, ApJ, 507, L59

Mandel, I., Haster, C.-J., Dominik, M., \& Belczynski, K. 2015, MNRAS, 450, L85

Margalit, B., \& Metzger, B. D. 2019, ApJ, 880, L15

Martin, D., Perego, A., Arcones, A., et al. 2015, ApJ, 813, 2

Metzger, B. D. 2017, Liv. Rev. Relativ., 20, 3

Metzger, B. D., \& Fernández, R. 2014, MNRAS, 441, 3444

Metzger, B. D., Martínez-Pinedo, G., Darbha, S., et al. 2010, MNRAS, 406 2650

Miller, M. C., Lamb, F. K., Dittmann, A. J., et al. 2019, ApJ, 887, L24

Özel, F., Psaltis, D., Narayan, R., \& McClintock, J. E. 2010, ApJ, 725, 1918

Özel, F., Psaltis, D., Narayan, R., \& Santos Villarreal, A. 2012, ApJ, 757, 55

Pannarale, F., Berti, E., Kyutoku, K., Lackey, B. D., \& Shibata, M. 2015a, Phys. Rev. D, 92, 084050

Pannarale, F., Berti, E., Kyutoku, K., Lackey, B. D., \& Shibata, M. 2015b, Phys. Rev. D, 92, 081504

Perego, A., Rosswog, S., Cabezón, R. M., et al. 2014, MNRAS, 443, 3134

Perego, A., Radice, D., \& Bernuzzi, S. 2017, ApJ, 850, L37

Pozanenko, A. S., Minaev, P. Y., Grebenev, S. A., \& Chelovekov, I. V. 2020 Astron. Lett., 45, 710

Radice, D., Perego, A., Hotokezaka, K., et al. 2018a, ApJ, 869, L35

Radice, D., Perego, A., Zappa, F., \& Bernuzzi, S. 2018b, ApJ, 852, L29

Radice, D., Perego, A., Hotokezaka, K., et al. 2018c, ApJ, 869, 130

Raithel, C. A., Özel, F., \& Psaltis, D. 2018, ApJ, 857, L23

Read, J. S., Lackey, B. D., Owen, B. J., \& Friedman, J. L. 2009, Phys. Rev. D, 79, 124032

Riley, T. E., Watts, A. L., Bogdanov, S., et al. 2019, ApJ, 887, L21

Rosswog, S. 2005, ApJ, 634, 1202

Salafia, O. S., \& Giacomazzo, B. 2021, A\&A, 645, A93

Shibata, M., \& Taniguchi, K. 2011, Liv. Rev. Relativ., 14, 6

Shibata, M., Kyutoku, K., Yamamoto, T., \& Taniguchi, K. 2009, Phys. Rev. D, 79, 044030

Siegel, D. M., \& Metzger, B. D. 2017, Phys. Rev. Lett., 119, 231102
Siegel, D. M., Ciolfi, R., \& Rezzolla, L. 2014, ApJ, 785, L6

Song, H.-R., Ai, S.-K., Wang, M.-H., et al. 2019, ApJ, 881, L40

Steiner, A. W., Hempel, M., \& Fischer, T. 2013, ApJ, 774, 17

The ENGRAVE Collaboration (Ackley, K., et al.) 2020, A\&A, 643, A113

The LIGO Scientific Collaboration \& the Virgo Collaboration 2010, Classical Quantum Gravity, 27, 173001

The LIGO Scientific Collaboration \& the Virgo Collaboration 2017a, Phys. Rev. Lett., 119, 161101

The LIGO Scientific Collaboration \& the Virgo Collaboration 2017b, ApJ, 850, L39

The LIGO Scientific Collaboration \& the Virgo Collaboration 2019a, ApJ, 882, L24

The LIGO Scientific Collaboration \& the Virgo Collaboration 2019b, Gamma-ray Coordinates Network Circulars, 25695

The LIGO Scientific Collaboration \& the Virgo Collaboration 2019c, Gamma-ray Coordinates Network Circulars, 26350

The LIGO Scientific Collaboration \& the Virgo Collaboration 2019d, Gamma-ray Coordinates Network Circulars, 25829

The LIGO Scientific Collaboration \& the Virgo Collaboration 2019e, Gamma-ray Coordinates Network Circulars, 25871

The LIGO Scientific Collaboration \& the Virgo Collaboration 2019f, Phys. Rev. $\mathrm{X}, 9,011001$

The LIGO Scientific Collaboration \& the Virgo Collaboration 2019g, Gamma-ray Coordinates Network Circulars, 24168

The LIGO Scientific Collaboration \& the Virgo Collaboration 2020, ApJ, 892, L3

Thompson, T. A., Kochanek, C. S., Stanek, K. Z., et al. 2019, Science, 366, 637

Tsokaros, A., Ruiz, M., Shapiro, S. L., Sun, L., \& Uryū, K. 2020, Phys. Rev. Lett., 124, 071101

Typel, S., Röpke, G., Klähn, T., Blaschke, D., \& Wolter, H. H. 2010, Phys. Rev. C, 81, 015803

Villar, V. A., Guillochon, J., Berger, E., et al. 2017, ApJ, 851, L21

Vincent, T., Foucart, F., Duez, M. D., et al. 2020, Phys. Rev. D, 101, 044053 


\section{Appendix A: Disc mass fitting formula for NSNS mergers}

\section{A.1. Derivation of a heuristic disc mass formula}

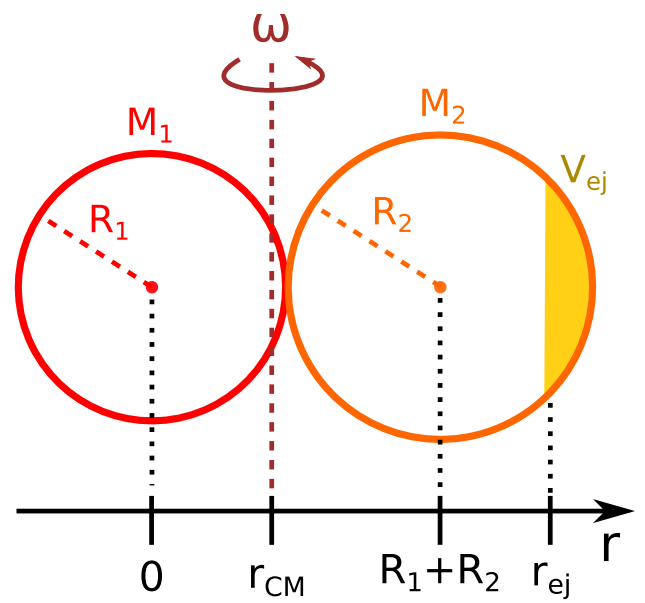

Fig. A.1. Sketch of the reference geometry in the toy model on which the disc mass fitting formula is based.

In asymmetric NSNS mergers, the main mechanism that leads to the formation of an accretion disc is the tidal disruption of one of the two NSs (Bernuzzi et al. 2020). To estimate the amount of tidally disrupted material that goes into forming the accretion disc, we look for the fraction of NS material that is centrifugally supported, at the onset of the merger, against falling directly into the remnant. We do not aim here at an accurate physical description of the process, but rather we use intuitive geometrical reasoning to get to an analytical form with some free parameters that can then be fitted to simulation data. We validate the formula a posteriori by assessing the scatter of the simulation data with respect to its predictions. Here we consider a NS binary of masses $M_{1}$ and $M_{2}$ and radii $R_{1}$ and $R_{2}$, right at the moment when the two surfaces touch each other (refer to Fig. A.1 for a sketch of the geometry). For the moment we will neglect the tidal deformation of the two stars as well as the relativistic effects. Assuming Keplerian orbits, the angular frequency of the binary is $\omega=\sqrt{G M /\left(R_{1}+R_{2}\right)^{3}}$, where $M=M_{1}+M_{2}$ is the total mass. If we set the origin of our coordinate system at the centre of $M_{1}$, then the centre of mass of the system is located at a distance $r_{\mathrm{CM}}=\left(R_{1}+R_{2}\right) /\left(1+M_{1} / M_{2}\right)$ along the line that connects the centres of the two stars. At any point $r>r_{\mathrm{CM}}$ along this line, the centrifugal acceleration experienced by

$a_{\mathrm{c}}=\frac{G M}{\left(R_{1}+R_{2}\right)^{2}} \frac{r / r_{\mathrm{CM}}-1}{1+M_{1} / M_{2}}$.

Now, our ansatz is that whenever this centrifugal acceleration exceeds the gravitational acceleration

$a_{\mathrm{g}}=\frac{G M}{\left(r-r_{\mathrm{CM}}\right)^{2}}=\frac{G M}{\left(R_{1}+R_{2}\right)^{2}} \frac{\left(1+M_{1} / M_{2}\right)^{2}}{\left(r / r_{\mathrm{CM}}-1\right)^{2}}$

that the merger remnant (assuming no mass loss) would exert at the same distance, then the corresponding part of the star $M_{2}$ can be centrifugally supported. If tidal forces cause the star $M_{2}$ to stretch to an ellipsoid whose semi-major axis is $\lambda_{2} R_{2}$, then the effect is roughly that of reducing $a_{\mathrm{g}}$ by $\lambda_{2}^{2}$ and increasing $a_{\mathrm{c}}$ by $\lambda_{2}$ at the corresponding position. By the condition $a_{\mathrm{g}} / \lambda_{2}^{2}<a_{\mathrm{c}} \lambda_{2}$, one obtains that matter beyond $r_{\mathrm{ej}, 2}=\left(R_{1}+R_{2}\right)\left[\left(1+M_{1} / M_{2}\right)^{-1}+\right.$ $\left.\lambda_{2}^{-1}\right]$ is centrifugally supported. The mass of this matter can be estimated by assuming the NS density profile to be uniform, and approximating the volume $V_{\mathrm{ej}}$ of the ejected matter as a spherical cap (which is reasonable as long as it is small compared to the sphere), which yields

$M_{\mathrm{d}, 2}=\frac{V_{\mathrm{ej}}}{V} M_{2} \sim \frac{1}{4}\left(2+x_{2}\right)\left(x_{2}-1\right)^{2} M_{2}$,

where $x_{2}=\left(r_{\mathrm{ej}, 2}-R_{1}-R_{2}\right) / R_{2}$ and we are neglecting the difference between baryon and gravitational mass and the fact that a small fraction of this mass could be unbound and contribute to the dynamical ejecta rather than to the accretion disc. If both components have masses not too close to the maximum TOV mass, this can be simplified further by neglecting the difference in NS radii. With this assumption,

$x_{2} \sim 2\left(\left(1+M_{1} / M_{2}\right)^{-1}+\lambda_{2}^{-1}-1\right)$,

and we impose $0 \leq x_{2} \leq 1$. Exchanging 1 and 2 , one gets the corresponding formula for the disc mass contribution $M_{\mathrm{d}, 1}$ from the star $M_{1}$, so that the disc mass is eventually $M_{\mathrm{d}}=M_{\mathrm{d}, 1}+M_{\mathrm{d}, 2}$.

\section{A.2. Fitting to simulation data}

In order to link the tidal deformability parameters $\lambda_{1,2}$ to quantities that can be measured from the GW signal, we make the following ansatz:

$\lambda_{1}=\left(\frac{\tilde{\Lambda}}{\Lambda_{0}}\right)^{\alpha}\left(\frac{M_{2}}{M_{1}}\right)^{\beta}, \quad \lambda_{2}=\left(\frac{\tilde{\Lambda}}{\Lambda_{0}}\right)^{\alpha}\left(\frac{M_{1}}{M_{2}}\right)^{\beta}$,

which encodes the fact that the lighter NS is more deformable than the heavier one. Here $\tilde{\Lambda}$ is the dimensionless tidal deformability parameter of the binary (Raithel et al. 2018). As a final tuning, we assume a floor disc mass of $M_{\mathrm{d}, \min }=10^{-3} M_{\odot}$ as in Radice et al. (2018a).

The fitting formula has three free parameters, namely $\Lambda_{0}, \alpha$ and $\beta$. We determine these parameters by least-squares fitting the logarithm of the disc masses predicted by the toy model to the results of the numerical simulations presented in Radice et al. (2018a), Kiuchi et al. (2019), Bernuzzi et al. (2020), and Vincent et al. (2020). We include all simulations reported in these works, despite some of them describing the same system but with differing setup (e.g. different treatments of neutrino transport): this has the effect of including, in a crude way, the modelling uncertainty. We obtain the best fit values $\Lambda_{0}=245, \alpha=0.097$ and $\beta=0.241$. The result is shown in Fig. A.2, where data points represent the disc masses as measured in the simulations, as a function of $\tilde{\Lambda}$. Squares, circles, upward-pointing triangles, and downwardpointing triangles are data from Bernuzzi et al. (2020), Kiuchi et al. (2019), Radice et al. (2018a), and Vincent et al. (2020), respectively. The error bars represent the uncertainty in the disc mass as defined in Radice et al. (2018a). The upper panel shows representative curves $M_{\mathrm{d}}(\tilde{\Lambda}, q)$ from our fitting formula, for $q \in$ $\{0.77,0.86,0.91,0.96,1\}$, assuming $M_{1}+M_{2}=3 M_{\odot}$ (for both data points and curves, the value of $q$ is colour-coded according to the colour bar on the right). The lower panel shows the relative residuals between model and data (in this case, the appropriate total mass $M_{1}+M_{2}$ for each data point is used). The relative residuals are below 0.5 for $68 \%$ of the simulations, and below 0.9 for $90 \%$ of them. We also note the close similarity between the equal-mass case (yellow line) and the fitting formula by Radice et al. (2018a) (black dashed line, shown for comparison). 


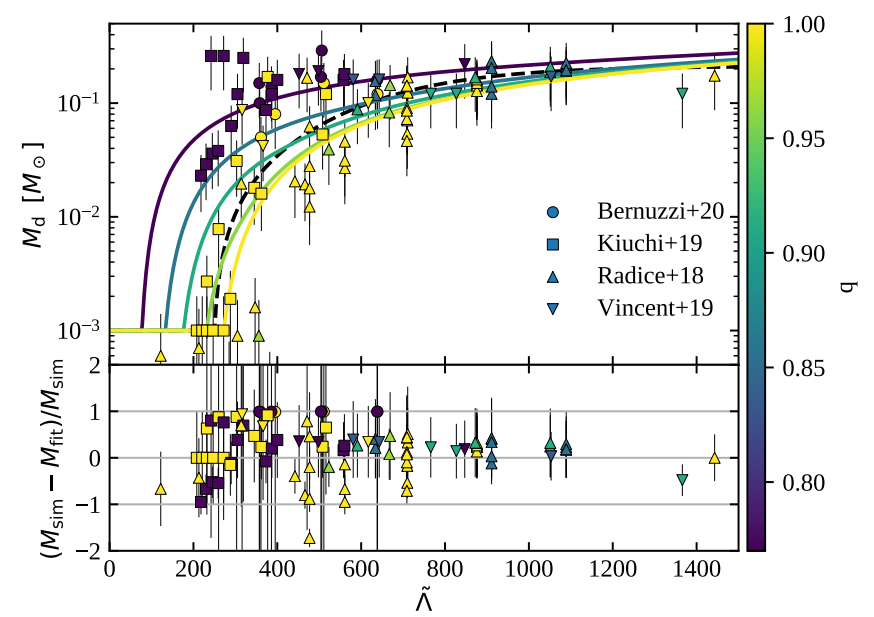

Fig. A.2. Comparison between disc masses from numerical relativity simulations and the predictions of our fitting formula (Eqs. A.3, A.4, and A.5). In both panels, data points show the disc masses reported in (Bernuzzi et al. 2020, squares), (Kiuchi et al. 2019, circles), (Radice et al. 2018a, upward-pointing triangles), and (Vincent et al. 2020, downward-pointing triangles) as a function of the dimensionless tidal deformability parameter $\tilde{\Lambda}$ of the corresponding NS binary. The colour of each marker shows the mass ratio $q$ of the binary, as coded in the colour bar on the right. In the upper panel, solid lines show the predictions of our fitting formula, assuming a representative total mass of $M_{1}+M_{2}=3 M_{\odot}$. The dashed black line shows the fit from Radice et al. (2018a) for comparison. The lower panel shows the relative residuals between the fitting formula (evaluated with the appropriate total mass for each binary) and the results from the simulation. More details are provided in the text.

\section{Appendix B: KN light curves varying model parameters}

In this section we perform the analysis presented in Sect. 4 considering some variations in the model parameters. The aim of this section is to test the robustness of our results and their sensitivity to modelling assumptions. We consider three variations. In Variation 1 (V1), as explained above, the NSNS configurations corresponding to GW190425- $M_{\mathrm{c}}$ involve mostly asymmetric binaries. Bernuzzi et al. (2020) recently found that asymmetric NSNS mergers produce dynamical ejecta with a crescent-like geometry, similarly to BHNS mergers (Kawaguchi et al. 2016). Thus in V1 we set the NSNS dynamical ejecta geometrical parameters to $\theta_{\mathrm{d}}=20 \mathrm{deg}$ and $\phi_{\mathrm{d}}=\pi \mathrm{rad}$. In Variation 2 (V2), besides being mostly asymmetric, NSNS configurations corresponding to GW190425- $M_{\mathrm{c}}$ also involve massive stars. As explained in Sect. 3, in almost all the cases the merger results in a prompt $\mathrm{BH}$ formation, without an intermediate hyper-massive NS phase. The consequent lack of neutrino winds (and neutrinomatter interaction) could lead to less massive wind ejecta with a smaller electron fraction (larger opacity). In such a scenario the ejecta properties would be similar to the BHNS case. Thus in V2 we set the NSNS parameters $\xi_{\mathrm{w}}, k_{\mathrm{w}}, \theta_{\mathrm{d}}$ and $\phi_{\mathrm{d}}$ to the same values of the BHNS case.

Finally, in Variation 3 (V3) we explore the case in which BHNS mergers produce ejecta with much larger opacities (lower electron fractions). We set $k_{\mathrm{d}}=30 \mathrm{~cm}^{2} / \mathrm{g}, k_{\mathrm{w}}=5 \mathrm{~cm}^{2} / \mathrm{g}$ and $k_{\mathrm{s}}=15 \mathrm{~cm}^{2} / \mathrm{g}$.

Figure B.1 shows the analogues of Fig. 5 for V1 (top row), V2 (central row), and V3 (bottom row). Concerning V1, we find that the different dynamical ejecta geometry does not affect the expected NSNS KN ranges for APR4 and only slightly changes the ranges for DD2. Indeed for the considered binary configurations, as shown in Fig. 2, for the APR4 EoS no dynamical ejecta are produced, while for DD2 they have small masses and the dominant components are the ejecta from the disc. Concerning $\mathrm{V} 2$, we find that the reduced wind ejecta mass produces dimmer light curves. Concerning V3, we find that increasing the BHNS ejecta opacities produce dimmer light curves (the ranges are shifted of $\sim 0.5 \mathrm{mag}$ ).

Therefore, we find that also for these three different model parameters variations our results remain valid. Indeed for V1 the ranges are very similar to Fig. 5, for V2 the overlap between BHNS and NSNS expected ranges is even smaller, while for V3 the overlap is a bit larger. However, in each case it remains possible to distinguish the nature of the merging ambiguous system through the observation of the KN produced by the merger. This demonstrates the robustness of our results with respect to different assumptions on model parameters. 
C. Barbieri et. al: Exploring the nature of ambiguous merging systems: GW190425 in low latency
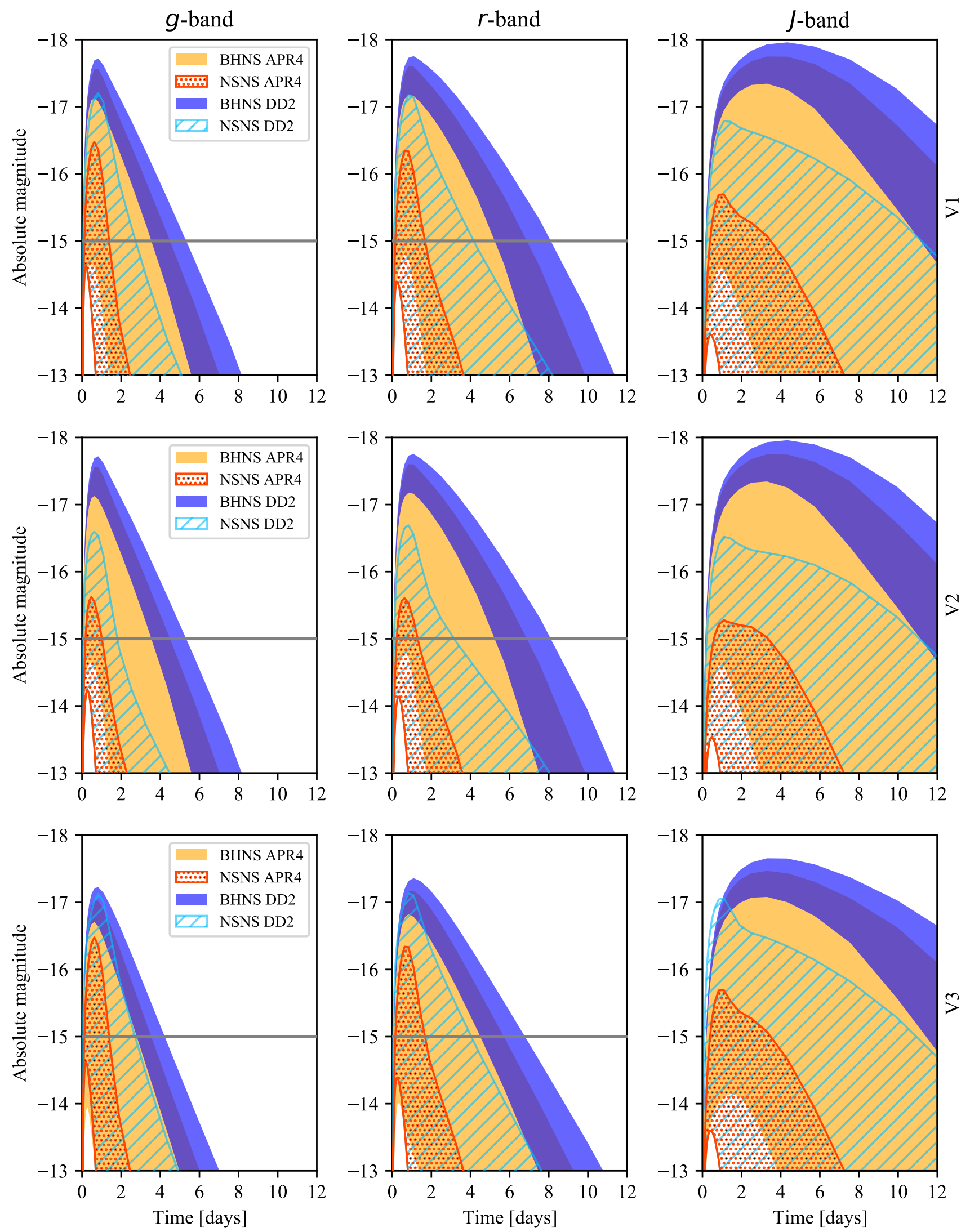

Fig. B.1. Same as Fig. 5, but for model parameter variations V1 (top row), V2 (central row), and V3 (bottom row). See Appendix B for a description of these variations. 